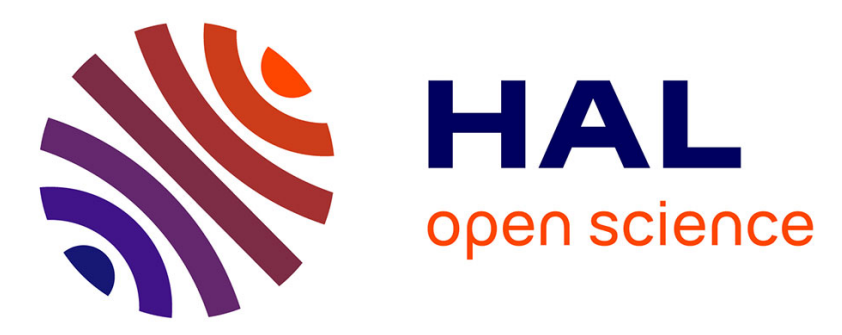

\title{
Thermocapillary instability of an ionic liquid -water mixture in a temperature gradient
}

Marc Pascual, Axelle Amon, Marie-Caroline Jullien

\section{To cite this version:}

Marc Pascual, Axelle Amon, Marie-Caroline Jullien. Thermocapillary instability of an ionic liquid -water mixture in a temperature gradient. Physical Review Fluids, 2021. hal-03235585

\section{HAL Id: hal-03235585 \\ https://hal.science/hal-03235585}

Submitted on 21 Nov 2021

HAL is a multi-disciplinary open access archive for the deposit and dissemination of scientific research documents, whether they are published or not. The documents may come from teaching and research institutions in France or abroad, or from public or private research centers.
L'archive ouverte pluridisciplinaire HAL, est destinée au dépôt et à la diffusion de documents scientifiques de niveau recherche, publiés ou non, émanant des établissements d'enseignement et de recherche français ou étrangers, des laboratoires publics ou privés. 


\title{
Thermocapillary instability of an ionic liquid - water mixture in a temperature gradient
}

\author{
Marc Pascual \\ Gulliver CNRS UMR 7083, PSL Research University, \\ ESPCI Paris, 10 rue Vauquelin, 75005 Paris, France and \\ Université Rennes 1, CNRS, IPR (Institut de Physique de Rennes) - UMR 6251, F-35000 Rennes, France \\ Axelle Amon \\ Université Rennes 1, CNRS, IPR (Institut de Physique de Rennes) - UMR 6251, F-35000 Rennes, France \\ Marie-Caroline Jullien* \\ Gulliver CNRS UMR 7083, PSL Research University, \\ ESPCI Paris, 10 rue Vauquelin, 75005 Paris, France and \\ Université Rennes 1, CNRS, IPR (Institut de Physique de Rennes) - UMR 6251, F-35000 Rennes, France
}

(Dated: November 21, 2021)

\begin{abstract}
Ionic liquids have remarkable properties and are commonly harnessed for green chemistry, lubrication and energy applications. In this paper, we study a thermo-responsive Ionic Liquid (IL) solution which has the property of phase separating above a critical temperature, an interesting feature for the recovery of the IL-rich phase. For this purpose, we generate a temperature gradient in a microfluidic cavity where the confinement strengthens wetting effects and enhances the demixing. In this experimental configuration, we report the separation patterns along the phase diagram of the binary mixture composition. Three separation dynamics regime are identified that may display complex three-dimensional flows. In spite of this complexity, we rationalize all the observed regimes. Only two regimes lead to a complete spatial separation of the two phases. Interestingly, one is reminiscent of a Marangoni instability in radial geometry, even at confinement below $100 \mu \mathrm{m}$. We believe this work will find applications in the recycling of ionic liquids.
\end{abstract}

\section{INTRODUCTION}

Since the 2010s, thermoresponsive binary systems made of water and ionic liquids (IL) have proved useful for several applications such as protein stabilisation [1-3], catalytic synthesis [4], metal and rare-earth extraction [5-7], forward osmosis water desalination [8-10] and low grade heat valorization [11, 12]. For all these techniques the temperature is used as a control parameter to trigger the phase separation and recover on demand the IL-rich phase of interest. This separation can be managed in bulk using commercial centrifugation techniques or more simply by letting the two liquids separate by density [13]. Yet ionic liquids are still costly and generally available in limited volumes, thus the aforementioned macroscopic techniques may not be adapted for the extraction of the IL-rich phase. For that purpose, microfluidics-based liquid-liquid separation is a promising alternative since wetting effects, which are marked in binary mixtures [14-16], are known to boost the phase-separation kinetics of binary systems [17-23].

It has been demonstrated that a confinement gradient helps to segregate two immiscible liquids according to their respective affinities for the walls of the cavity: the most wetting phase is dragged toward the wedge, i.e. toward the thinner part of the cavity [24]. Another possibility lies in the temperature-induced interfacial tension gradients, which are known to cause an interfacial stress generating thin film flow [25-28] and droplets or colloids motion [29-36], thereby affecting the phase separation pattern, as demonstrated by Beysens et. al. in 2002 with pure $\mathrm{CO}_{2}$ [37] [38]. More recently, we showed with a binary mixture how the use of a temperature gradient allows to selectively accumulate the entire wetting phase at the warmer side of the cavity by taking advantage of Marangoni effects at small scales [39].

In this paper, by heating locally the center of a cylindrical cavity, we report the separation patterns along the phase diagram of a binary mixture composition. More precisely, using a mixture composed of water and ionic liquid, the coupling of Marangoni strains and selective wetting leads to three caracteristic separation patterns. As for the dynamics of binary mixtures under homogeneous temperature, the initial composition of the solution imposes the phase separation pattern $[40,41]$. Each pattern is obtained for a certain range of composition and interestingly two of them yield a selective accumulation of the IL-rich phase at the hotter part of the cavity. We present successively the three separation patterns in ascending order of IL mass fraction. The first regime, in which water is the main component, has been reported and rationalized in a previous work and for sake of clarity the main mechanisms are briefly recalled [39]. The second regime, beyond the consolute point of the mixture, generates an unexpected 
Bénard-Marangoni-like periodic pattern. We interprate this regime as a thermocapillary driven instability, whose mechanisms involved are actually different from the classical Bénard-Marangoni instability. This regime is analyzed in details and constitutes the main part of the paper. The third regime, far beyond the consolute point of the mixture, is quickly explained as it presents less interest for the selective separation. In the end we discuss the pertinence of the three regimes for IL recovery applications.

MATERIAL \& METHODS

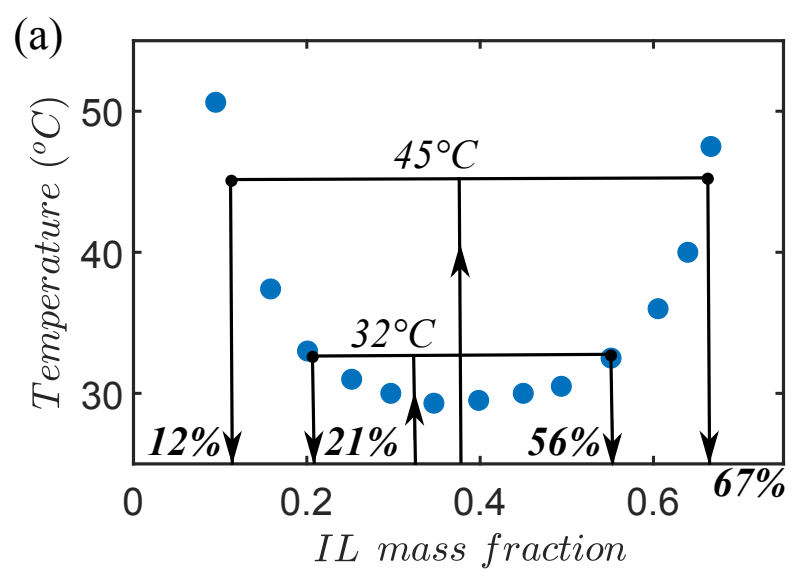

(b)

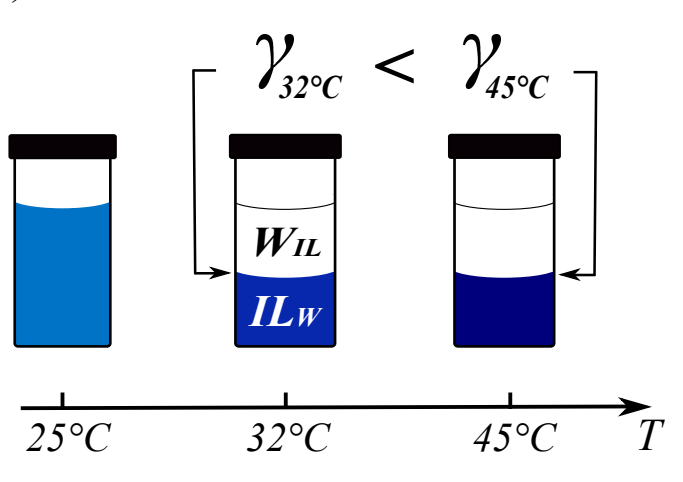

FIG. 1. (a) Phase diagram of the IL-water mixture. The weight fractions in IL of both water-rich and IL-rich phases obtained after separation at $32^{\circ} \mathrm{C}$ and $45^{\circ} \mathrm{C}$ are indicated. (b) Sketch of the binary mixtures samples in bulk at different temperatures. The interfacial tension $\gamma$ between the two phases increases with the temperature as our system is LCST. $I L_{W}$ indicates the IL-rich phase, $W_{I L}$ the water-rich phase. The IL-rich phase is dyed in blue.

The thermosensitive binary system of interest is a mixture of MilliQ water with the ionic liquid tetrabutylphosphonium trifluoroacetate $\left(\mathrm{P}_{4444} \mathrm{CF}_{3} \mathrm{CO}_{2}\right)$ [42, 43] already used in different applications [9-11], at an IL mass fraction comprised between $15 \%$ and $60 \%$ (separation temperature $T_{\text {sep }}=30$ to $39^{\circ} \mathrm{C}$ ). This system is known as a Lower Critical Solution Temperature (LCST) binary solution, i.e. it phase separates above a critical temperature. Figure 1(a) displays the phase diagram of this binary mixture. In order to understand how flows can be generated by a temperature gradient in such a binary system, it is necessary to understand the physico-chemical response of the system to two different temperatures $T_{1}<T_{2}$, see figure 1 . At the temperature $T_{1}$, e.g. $T_{1}=32^{\circ} \mathrm{C}$, the binary system is composed of two phases, one rich in ionic liquid with a mass fraction of $56 \%$ and the other rich in water with a mass fraction in ionic liquid of $21 \%$. We thus obtain two phases more or less rich in ionic liquid, but not pure in either of the two components. The surface tension between these two solutions is noted $\gamma_{1}$. Let us now consider that the temperature of the system is increased to $T_{2}$, e.g. $T_{2}=45{ }^{\circ} \mathrm{C}$. At this temperature, the binary system is composed of a phase rich in ionic liquid with a mass fraction of $67 \%$ and the other phase rich in water has a mass fraction in ionic liquid of $12 \%$. Thus the two phases are now respectively richer in ionic liquid and in water. This results in a surface tension $\gamma_{2}>\gamma_{1}$. The interfacial tension of LCST mixtures thus increases with temperature, see figure 2(c). Note that the surface tension is 0 at the consolute point, see figure 2(c). Such interface placed in a temperature gradient generates a Marangoni stress $\partial \gamma / \partial \tau$, where $\tau$ is the dimensional unit element along the tangent to the interface, oriented from cold to hot.

The microfluidic system that is used to generate a temperature gradient is sketched in Figure 2(a). The polydimethylsiloxane (PDMS) cavity is replicated from a micromilled brass mold, and bonded on a glass coverslip with oxygen plasma treatment. The most wetting phase is the IL-rich phase, an important consequence in the following is that the IL-rich phase can form a wetting film while the water phase will display non-wetting droplets. The cavity is a cylinder of diameter $1 \mathrm{~cm}$ whose height ranges from 20 to $200 \mu \mathrm{m}$. The bottom surface is sealed onto a glass wafer with micropatterned resistance $\left(50 \times 500 \mu \mathrm{m}^{2}\right)$, electrically isolated by spincoating a $30 \mu \mathrm{m}$ PDMS layer [44]. The potential applied to the heating resistor can be tuned up to 10 Volts. The temperature profile generated by the local resistance is isotropic and its radial dependency is shown in figure 2(b), the methodology allowing to measure this profile is reported in our previous work [39]. This gradient is well modelled by a $1 / \mathrm{r}$ decrease as shown in insert of 

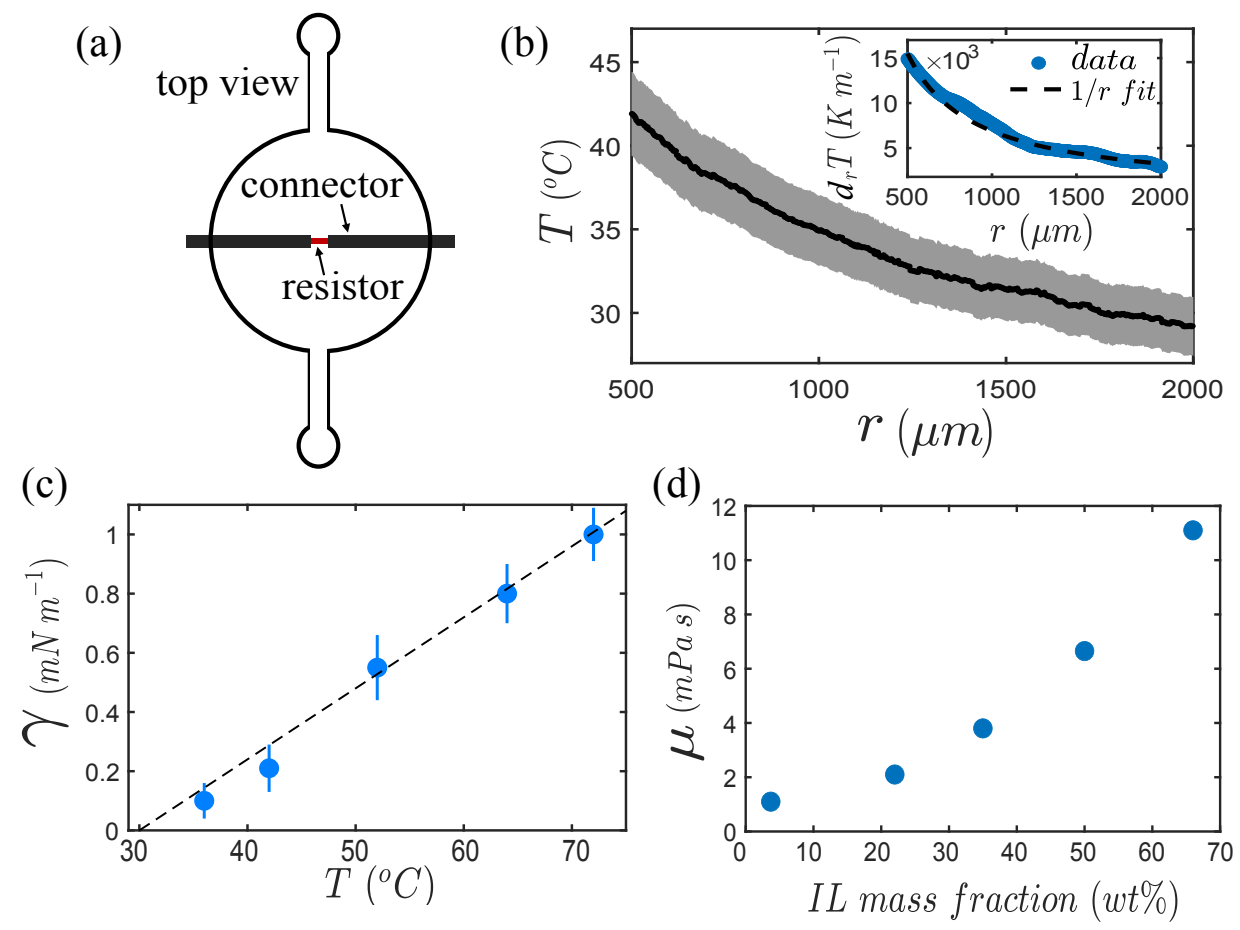

FIG. 2. (a) Top view of the microfluidic cavity $1 \mathrm{~cm}$ in diameter. The gold connectors are in dark grey, and the chromium resistor in red $\left(50 \times 500 \mu \mathrm{m}^{2}\right)$. (b) Radial cut of the temperature profile in the cavity around $t=70 \mathrm{~s}$. Inset: temperature gradient derived from the temperature profile fitted with $1 / r$. (c) Interfacial tension between the two separated phases versus temperature, measured by rising drop method. (d) Viscosity of the IL solution as a function of its concentration at $25^{\circ} \mathrm{C}$.

Figure 2(b). By increasing locally the temperature, we expect the mixture to phase separate, whose consequence is the generation of interfaces. Placed in a temperature gradient, these interfaces will be subjected to an interfacial stress proportional to the temperature gradient. Thus, the thermocapillary stress is radial, directed toward the warmer region, i.e. toward the center of the cavity. Finally, the last physicochemical parameter characterizing the system is the viscosity which is displayed as a function of the IL mass fraction on Figure $2(\mathrm{~d})$ at $25^{\circ} \mathrm{C}$.

Once the voltage is applied, the temperature suddenly increases at the resistor location and diffuses radially along the cavity. This diffusion triggers the phase separation which propagates from the center towards the edges, which is observed using bright-field transmitted light video microscopy. The signature of the separation temperature $\left(T_{\text {sep }}\right)$ isotherm can be captured typically on figures 4 and 5 , at the front of the dark cloud stemming from the light scattering of the dense two phase system, whose droplets sizes are much smaller than the cavity thickness. The temperature steady state is reached after 5 to 10 minutes depending on room temperature.

\section{PATTERNS OF SEPARATION}

By scanning the dynamics of separation as a function of composition, different patterns are observed, that can be brought together in three different regimes summarized in Figure 3. The dynamics of these three regimes are qualitatively described below, see Supplemental Movie 1 showing the phase separation in both three regimes [46].

- Below $35 \mathrm{wt} \%$ of IL (consolute point), the dynamics is associated with a complex three-dimensional flow generated by the combination of thermocapillary effects and sedimentation (see regime A on Fig. 3 and supplementary movie 1). This regime was published in [39] and is summarized in section IV.

- Between $35 \mathrm{wt} \%$ and $55 \mathrm{wt} \%$, the flow turns to an instability pattern driven by thermocapillarity (see regime B on Fig. 3 and supplementary movie 1). This regime is rationalized using a linear stability analysis in section $\mathrm{V}$ and is the core of the paper. 
- Above $\sim 55 \mathrm{wt} \%$, the solution is composed essentially of ionic liquid. There is therefore a dispersed phase of water in ionic liquid in the form of drops which are expelled outwards by thermocapillary migration (see regime $\mathrm{C}$ on Fig. 3 and supplementary movie 1). This regime is described in section VI.

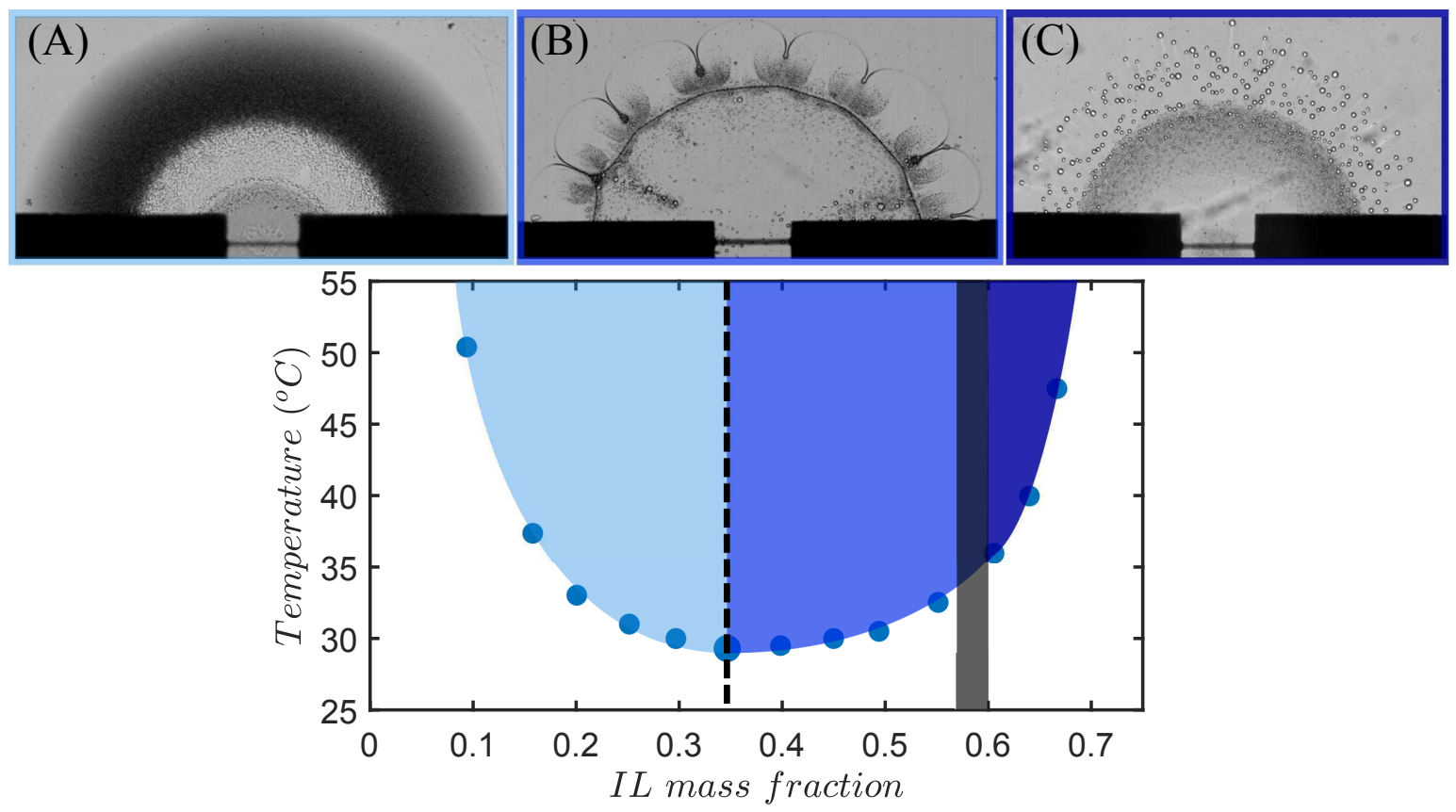

FIG. 3. Phase diagram of the IL - water mixture with the three phase separation patterns observed experimentally. The transition from first to second regime is indicated by the vertical dashed line centered on the consolute point. These two regimes selectively accumulate the wetting phase (IL-rich here) above the heating resistor. The transition from second to third regime is given by the dark grey area, when the composition is between 55 and $60 w t \%$ in IL. The third regime does not yield a selective separation.

The transition from regime 1 to 2 is governed by the transition of composition at the critical point of the binary mixture at $35 \mathrm{wt} \%$ in ionic liquid. On the left of this point, i.e. for lower ionic liquid mass fraction, the emulsion is composed of IL droplets into water and water is the continuous phase. On the right of this point, i.e. for higher IL mass fraction, the emulsion is inversed, we thus obtain water droplets and the ionic liquid is the continuous phase. Since both phases have similar dynamical properties, the separation line between the two demixing regimes is almost vertical [45].

Transition from regime 2 to 3 is less obvious because in both cases water droplets are produced in a continuous ionic liquid solution. We notice experimentally a transition of the separation pattern from a flower shape to a fireworks-like around 55-60 wt\% in ionic liquid. Qualitatively, this corresponds to the transition from a regime within which water droplets coalescence are observed, to a regime in which such events are not observed anymore. This is in agreement with what is observed in static experiments for which there is no coalescence events above this concentration [41].

In the prospect of separating a water-rich phase and a phase rich in ionic liquid, only the first two regimes were able to achieve this objective.

\section{IONIC LIQUID MASS FRACTION BELOW 35\%}

For sake of clarity, we describe shortly in this section the first separation regime already reported in [39]. This separation pattern is witnessed for an IL mass fraction range of 10 to $35 \mathrm{wt} \%$, i.e. when the IL phase is the minority phase in volume. Hence, during the separation the IL-rich phase is the dispersed phase while the water-rich phase is the continuous phase.

Figure 4(a) displays a temporal evolution of the separation pattern. When the heating resistor is switched on, the temperature increases in the cavity and expands radially producing an opaque cloud of IL-rich droplets. The front of this cloud indicates the position of the phase separation isotherm $\left(31^{\circ} \mathrm{C}\right.$ at $\left.25 w t \%\right)$. Surprisingly, the cloud opens 


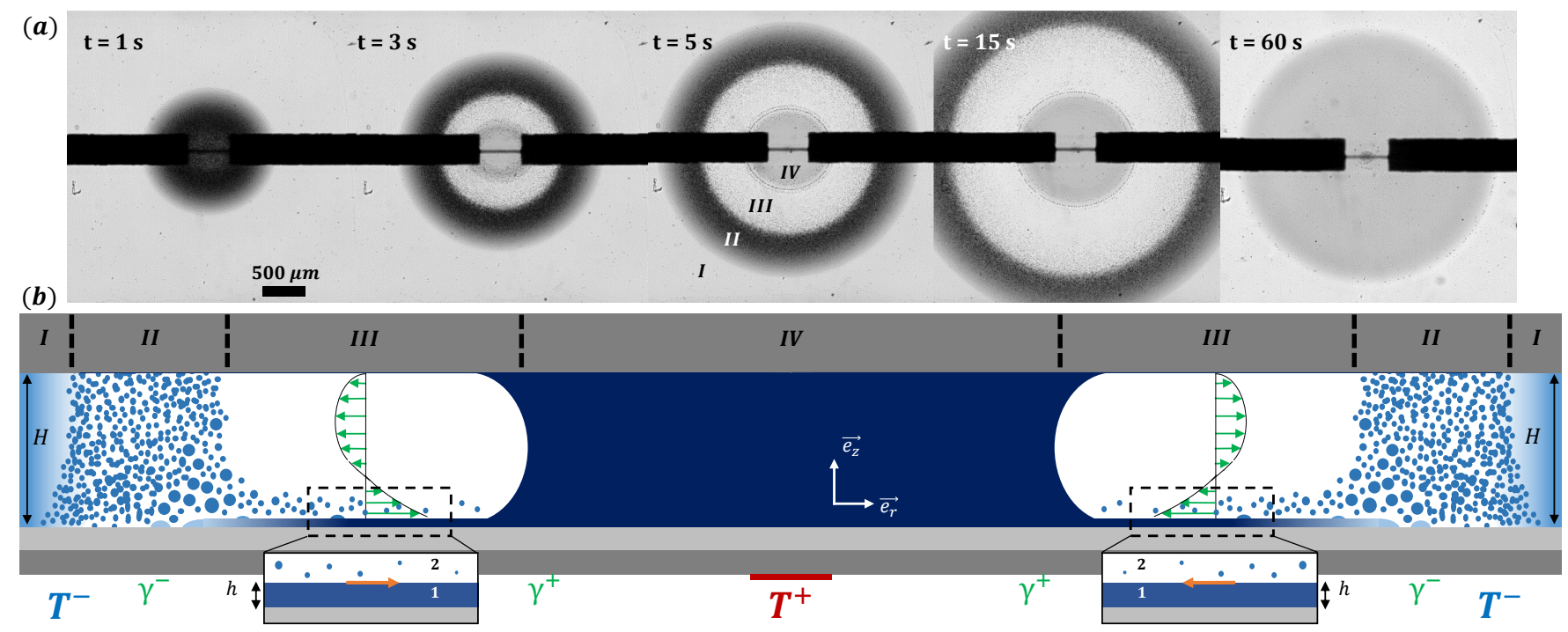

FIG. 4. (a) Top view of the separation dynamics at 7 Volts $(80 \mathrm{~mW})$ at different times. The mass fraction in IL is $25 \%$ and the cavity height is $H=60 \mu \mathrm{m}$. The separation dynamics can be divided into four regions, clearly visible at $t=5 \mathrm{~s}$ in the top view: I) IL-water non-separated mixture, II) separation of the two phases, the droplets are mostly pushed outwards, III) some IL-rich droplets are dragged inward across the water-rich phase, IV) IL-rich sessile drop. At $t=60 \mathrm{~s}$ when steady-state is reached, an IL-rich drop is accumulated above the heating resistor. (b) Cross-section sketch of the phase separation dynamics. The tread-milling wetting film induces a radial Couette-Poiseuille flow. Inset: within region III, domain 1 indicates the IL-rich wetting film and domain 2 the water-rich phase above. The orange arrows indicate the interfacial stresses.

up in its center to reveal an IL-rich drop. Moreover, a fraction of droplets emanating from the cloud are coming back to the center in an accelerated radial motion. This behavior results from the existence of a micrometer-thick wetting film covering the bottom of the cavity, as sketched on Figure 4(b). This film is a consequence of the sedimentation from the cloud's droplets (the IL is denser than water) which spread completely when impacting the bottom wall. The free interface of the film is submitted to thermocapillary stresses directed toward the center of the cavity, hence the IL within the film is drained toward the center where it accumulates to produce a bigger drop. Above the film the momentum diffusion drags along the water-rich phase and the neighbouring droplets from the cloud. The water phase converging to the center is sent back outwards by mass conservation and pushes away the cloud of droplets. This complexe three-dimensional flow has been rationalized and reported in [39]. In the end we recover an IL-rich drop in the center of the cavity surrounded by a water-rich liquid as seen at $t=60 \mathrm{~s}$ on figure 4 (a).

\section{IONIC LIQUID MASS FRACTION INBETWEEN 35\% AND 55\%}

\section{Description of the phase separation pattern}

Beyond the consolute point (35 wt\% in IL), the IL-rich phase becomes the continuous phase while the water-rich phase is the dispersed one. Figure 5 represents the typical dynamics of phase separation. At early time water droplets nucleate in the center and form an opaque cloud that expands isotropically in the horizonthal plane with the $T_{s e p}$ isotherm diffusion. These water droplets grow under coalescence events and are submitted to two moving mechanisms both driving the droplets outward : thermocapillary and thermomechanical migration. We recall briefly that the thermomechanical effect is associated to the propulsion of droplets, squeezed by both the bottom and top walls of a cavity, towards the cooler region due to a thinning of the cavity height in the warmer region [47]. The pattern of figure 5 is also observed in additional experiments performed in glass microcavities, in which the thermomechanical deformation is negligible. This observation thus allows us to disregard the thermomechanical mechanism in the following. Once the droplets reach the $T_{s e p}$ isotherm, their migration ceases and they sit on the isotherm circular line forming a flower-like pattern, recalling similar experiments involving Marangoni effects [48-52]. This crown of non-wetting water-rich drops delimits a central wetting drop enriched in Ionic Liquid. When steady state is reached, we end up with an IL-rich drop in the hot central region surrounded by a water-rich phase, similarly as in previous section. 


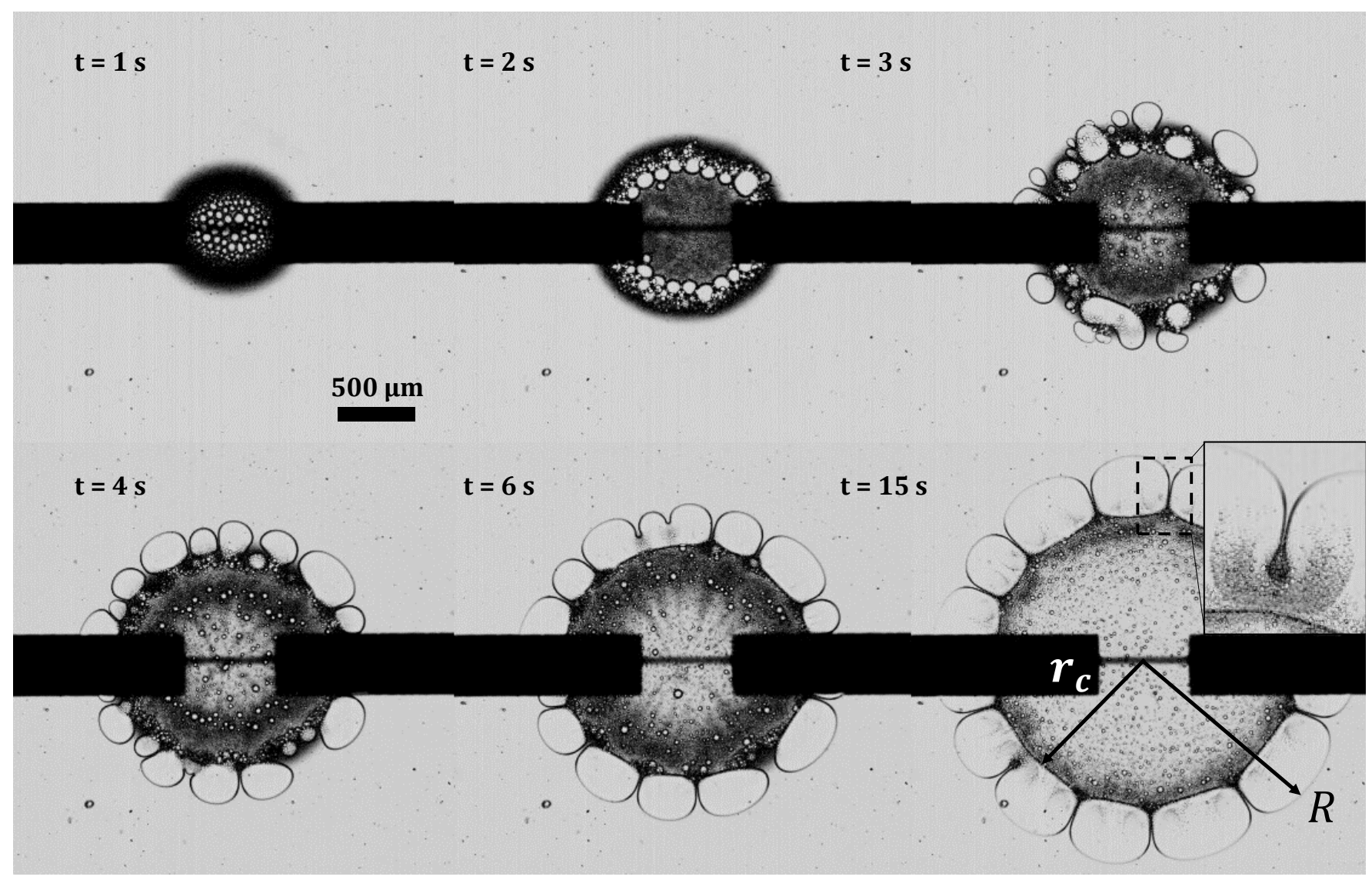

FIG. 5. Time lapse of the IL-water phase separation for 7 Volts $(80 \mathrm{~mW})$. The IL mass fraction is $45 \%$ and the cavity height is $H=80 \mu \mathrm{m}$. Water droplets nucleate, coalesce and get expelled outward. Once they cross the separation temperature isotherm (spotted as the outer radius of the darker circular zone) they stop and sit still on the periphery forming a crown of drops. This crown is delimited by its inner radius $r_{c}$ and its outer diameter $R$. Due to high enough water fraction, those drops remain side by side and their adjoining interfaces oriented radially are submitted to thermal interfacial gradients. Thus, these interfaces drain the outer liquid inward, making the central drop (rich in IL) to expand over time.

The initial shape of the flower-like pattern is governed by the initial water droplets that form the external crown. Inbetween drops, the liquid is drained from the outer unseparated region towards the center IL-rich region, and its gradual opacification is a result of the phase separation. We call those fingers "Marangoni jets", as we assume their active pumping coming from the thermocapillary stress sustains the expansion of the pattern. Inside the water drops, this flow induces convection cells that are made visible by the swirling plumes of small IL-rich droplets produced by the double phase-separation (see inset of Figure 5 at $t=15 \mathrm{~s}$ ) [53,54]. We believe that the very low interfacial tension inherent to near-critical binary mixtures is a key to the formation of such pattern [55-57].

Interestingly, the initial flower-like pattern evolves thanks to the spontaneous formation of jets during the expansion of the global pattern thus increasing the total number of petals. These jets usually appear when the inter-jet distance is much bigger than the recirculating plumes width (in other words when the water drop's azimuthal extent is at least twice as big as the jets length), see Figure 6 and Supplemental Movie 2 for a movie showing the spontaneous generation of new jets [58]. We believe that this digitation mechanism plays a major role in the regularity of the pattern. This observation leads us to rationalize this problem through an instability approach. After discussing the instability mechanism we propose two approaches to model this instability. First, we derive a scaling analysis that recovers the general trend of wavelength as a function the geometrical and physico-chemical parameters. In a second approach, a stability analysis inspired by the work of Nagel and Gallaire [59] is performed.

In order to look at the effect of wetting properties, additional measurements are made with a lutidine-water mixture which is also an LCST system. We recover the same demixing patterns proving that this behavior is not specific to ionic liquids. More details are given in Appendix A. 


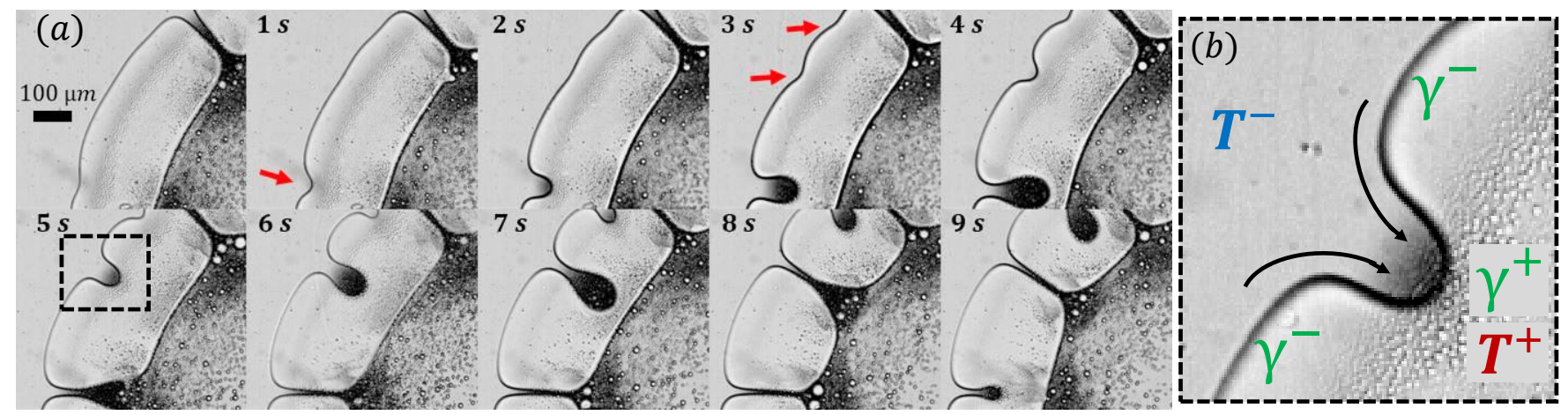

FIG. 6. (a) Spontaneous formation of Marangoni jets resulting from the thermocapillary runaway of small instabilities developping on the outer interface of the water drops. The initial positions of the perturbations are indicated by red arrows. (b) Zoom on the black dashed box of the picture $t=5 \mathrm{~s}$. The temperature gradient as well as the interfacial tension gradient are indicated along the finger interface.

\section{Instability mechanism}

We consider first the interface formed by the water-rich phase with the binary mixture placed at $r=R$. For a perfectly circular interface, the temperature field being radial, the surface tension is constant along the interface and is therefore stable. Let us now consider a radial fluctuation of this interface such that $r^{\prime}=R-\delta r$. The temperature is higher in $r^{\prime}$ than in $r$, and so is the surface tension. This results in a surface tension gradient $\delta \gamma=\gamma^{+}-\gamma^{-}$oriented from the larger $r$ to the smaller $r$, see figure 6(b). In this way, the fluctuation is amplified and leads to the formation of what we have called the Marangoni jets. By conservation of momentum, these jets generate a flow within the water phase, visible as recirculating zones in inset of Figure 5. The driving mechanism is thus mediated by the viscous dissipation in the water phase.

At first sight, it could be legitimate to associate this pattern to the Bénard-Marangoni instability. This instability is due to temperature fluctuations of the interface, at a given constant radius, which is maintained by the generated interfacial flow. The existence of this instability is in general conditioned by the Marangoni number which describes the ratio between the thermal diffusion time $t_{\kappa}=d^{2} / \kappa$, where $d$ is a characteristic length scale, and a transport time linked to the Marangoni stress $t_{M}=\mu /(\partial \gamma / \partial r)$. The instability is generally observed for Marangoni numbers $M a \gtrsim 10^{2}[?]$. The difference with our experiment is that our Marangoni number is small, $M a \lesssim 20$, so the temperature fluctuations at a given $r$ are negligible. Strictly speaking, what we observe is not a Bénard-Marangoni instability. Indeed, in our system it is a radial flow stemming from the interface radius fluctuations, thus exploring isothermal lines, that are amplified by the Marangoni stress, and not an orthoradial one as it would be the case in a Bénard-Marangoni instability. The fact that diffusion of heat does not come into play is reinforced by comparing the transport of heat by advection and diffusion, i.e. by estimating the thermal Péclet number in the water phase given by $P e=\left(\left(R-r_{c}\right) v_{r}\right) / \kappa \lesssim 0.5$ with $R-r_{c} \lesssim 500 \mu \mathrm{m}$ the characteristic crown extent, $\kappa=1.5 \cdot 10^{-7} \mathrm{~m}^{2} . \mathrm{s}^{-1}$ the water thermal conductivity and $v_{r}=150 \mu \mathrm{m} \cdot \mathrm{s}^{-1}$ the typical fluid velocity induced by thermocapillary forces [39]. As such, the temperature profile in a cross-section of the cavity is thus little affected by the flow. Consequently, the temperature fluctuations will not be considered in the following as we consider that heat is released faster by diffusion than by advection.

\section{Wavelength of the pattern}

In this subsection we study the influence of both the geometrical and the physico-chemical parameters on the pattern's wavelength and extension. We propose a scaling analysis rationalizing the observed dependency of the pattern wavelength.

Figure 7 displays snapshots of the phase separation pattern at $t=70 \mathrm{~s}$ for three cavity heights and three compositions. Qualitatively, the wavelength seems to increase with the thickness of the cavity at a given composition; on the other hand, it decreases when the composition increases in ionic liquid at a given cavity height. At $20 \mu \mathrm{m}$, we see that the patterns begin to be less well defined. This probably results from the important confinement which may 


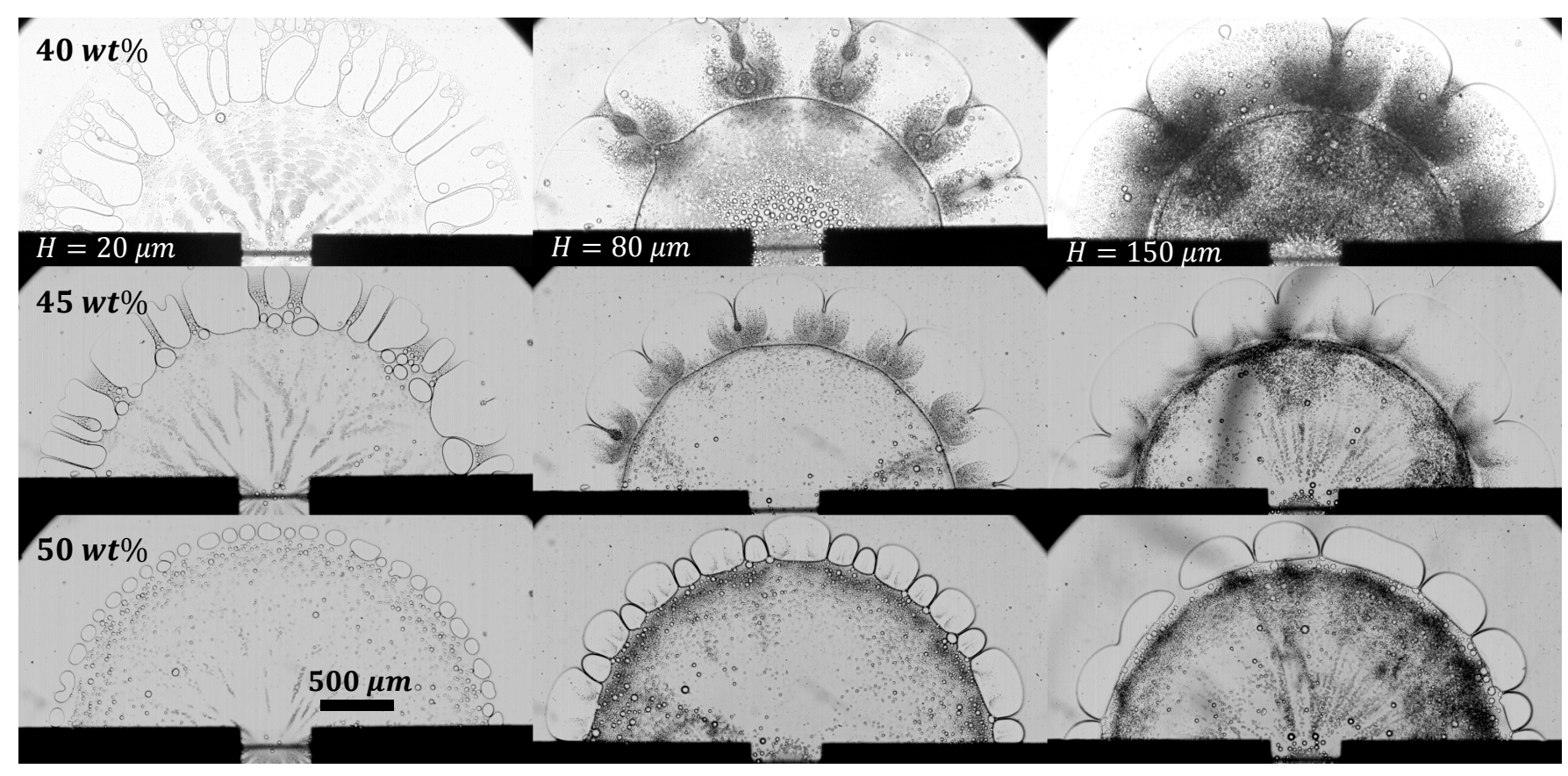

FIG. 7. Phase separation pattern at $t=50 \mathrm{~s}$, for three heights of cavity $H=20,80$, and $150 \mu \mathrm{m}$ (from left to right) and three compositions 40, 45 and $50 w t \%$ (from top to bottom). The voltage is 7 Volts $(80 \mathrm{~mW})$.

limits the velocity field and promote the stability of the filament, as evidenced by the absence of recirculation in the drops forming the crown. For the other confinements, the Marangoni jets show a well-defined periodic distribution which depends at a given time on the cavity height $H$ and on the mixture composition which signature is the crown extent $R-r_{c}$.

The number of jets $n_{j}$ are plotted as a function of $1 / H$ in Figure $8($ a) and displays a linear-like behavior (excluding the data at $H=20 \mu \mathrm{m})$, for the different compositions. More interestingly all the data collapse on a single curve by plotting the cell wavelength $\lambda \propto 1 / n_{j}$ as a function of the cross-section of the pattern $H \times\left(R-r_{c}\right)$. We now propose a qualitative description of this instability in order to recover this dependency, based on a similar approach as described in [60]. First, due to the confinement, two length scales have to be considered: $H$ and $R-r_{c}$. The destabilizing thermocapillary action across a cell writes as a force per unit length:

$$
F_{\gamma} \approx \frac{d \gamma}{d r}\left(R-r_{c}\right)
$$

This force is mediated by a stabilizing one stemming from viscous dissipation along the smallest lengthscale of the cavity: the height $H$. Hence, introducing $\lambda$ the wavelength instability (azimuthal length of a recirculating cell), the viscous force per unit length writes:

$$
F_{\mu}=\mu_{1} \frac{v_{\theta}}{H^{2}}\left(R-r_{c}\right) \lambda
$$

Incompressibility of the flow yields $v_{\theta}\left(R-r_{c}\right) \propto v_{r} \lambda$, and using the thermal Péclet number, this leads to:

$$
F_{\mu}=\mu_{1} \frac{\kappa P e \lambda^{2}}{H^{2}\left(R-r_{c}\right)}
$$

The threshold of the instability is estimated at $F_{\gamma} \gtrsim F_{\mu}$, yielding:

$$
\lambda^{2} \propto \frac{1}{\mu_{1} \kappa P e} \frac{d \gamma}{d r}\left(R-r_{c}\right)^{2} H^{2}
$$

This simple analysis allows to recover the correct scalings. The experiments using lutidine confirm this approach as shown by the black cross and black star on Figure 8 collapsing on the same curve. We will now check the order of magnitude of the prefactor of this scaling law. Equation 4 can be written in term of $n_{j}$ : 

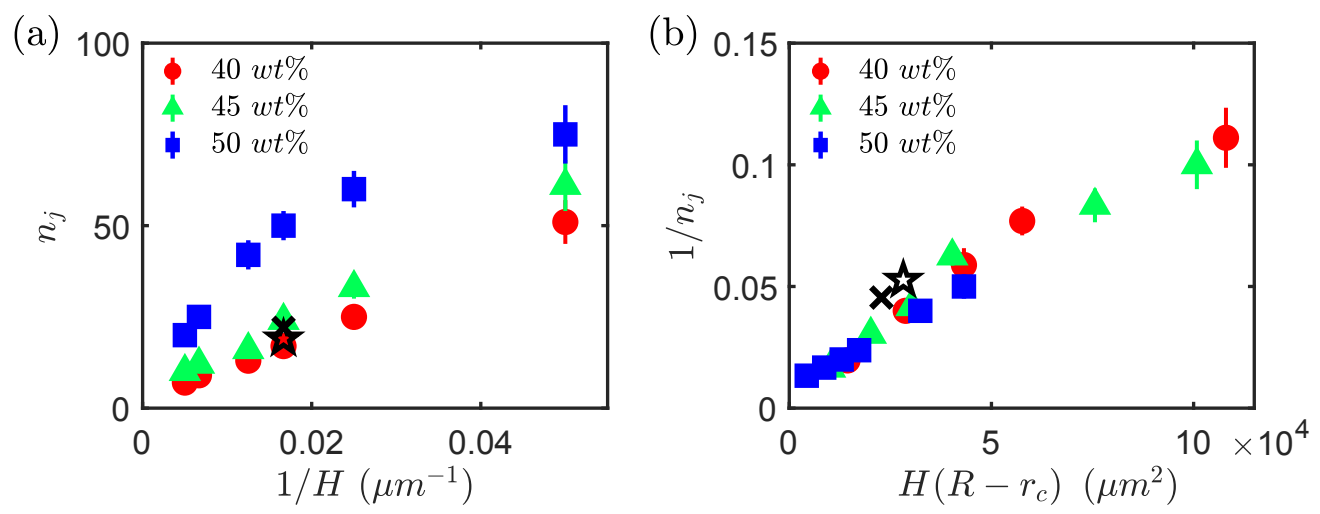

FIG. 8. (a) Number of jets $n_{j}$ measured at $t=70 \mathrm{~s}$ for six heights $H=20,40,60,80,150,200 \mu \mathrm{m}$ and three compositions: 40, 45 and $50 w t \%$ in ionic liquid. The dark cross and the dark star symbols are the number of jets from the lutidine-water experiments (see Figure 12 in Appendix A). (b) $1 / n_{j}$ (proportional to the wavelength) versus $H\left(R-r_{c}\right)$.

$$
\frac{1}{n_{j}} \propto \frac{1}{2 \pi R}\left(\frac{1}{\mu_{1} \kappa P e} \frac{d \gamma}{d r}\right)^{1 / 2}\left(R-r_{c}\right) H
$$

Assuming that $R=1,5.10^{-3} \mathrm{~m}, \mu_{1}=1.10^{-3}$ Pa.s, $\kappa=1,5.10^{-7} \mathrm{~m}^{2} \cdot \mathrm{s}^{-1}$ and $d \gamma / d r=d \gamma / d T \times d T / d r \approx 2.10^{-1}$ N.m ${ }^{-2}$, the prefactor of equation 5 is of order:

$$
\frac{1}{2 \pi R}\left(\frac{1}{\mu_{1} \kappa P e} \frac{d \gamma}{d r}\right)^{1 / 2} \sim 6.10^{-6} \mu \mathrm{m}^{-2}
$$

This value is of the same order than the slope obtained from figure 8 (b) of value $10^{-6} \mu \mathrm{m}^{-2}$. This fair agreement between our scaling and the experimental observations makes one confident in the analysis that has been done.

\section{Thermocapillary driven fingering instability}

In this section, we present an analytical model for the growth rate of the thermocapillary instability. Rather than using a Darcy-type approach typical of these confined systems, we have been inspired by the work of Nagel and Gallaire based on Brinkman's approach which takes into account in-plane shear stress [59]. The main difference between their model and our situation is that we replace a bulk driving mechanism by an interfacial driving mechanism (thermocapillary pumping).

A sinusoidal perturbation of the interface is considered, which is amplified by the radial interfacial tension gradients (see Figure 9). A linear stability analysis is performed in polar coordinates $\left(O, \overrightarrow{e_{r}}, \overrightarrow{e_{\theta}}, \overrightarrow{e_{z}}\right)$. We assume that the liquid composition is at equilibrium regarding the temperature at all time and that the volume variation due to the phase transition is negligible. The full calculation is provided in Appendix B, only the main steps and results are provided in this section.

The interface position is given by $R(\theta, t)$ and the sinusoidal perturbation writes:

$$
R(\theta, t)=R_{0}(t)+\epsilon \zeta(\theta, t)
$$

where

$$
\zeta(\theta, t)=\zeta_{0} e^{\beta t} e^{i n \theta}
$$

with $\beta$ the growth rate that depends on $n_{j}$. In practice, $R_{0}$ varies slowly in time compared to $\zeta$.

The interfacial tension depends on the perturbation as follow:

$$
\gamma=\gamma_{0}+\alpha \zeta
$$

with $\alpha=\frac{d \gamma}{d T} \frac{d T}{d r}<0$ the interfacial tension gradient. $\gamma_{0} \approx 10^{-4} \mathrm{~N} \cdot \mathrm{m}^{-1}$. The value of $\gamma_{0}$ is estimated from our interfacial tension measurements from Figure 2(d). However, we acknowledge that the position of the interface in 


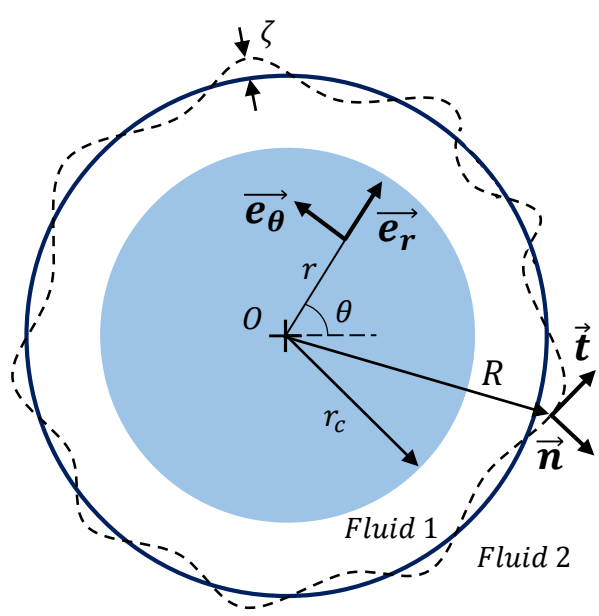

FIG. 9. Sinusoidal perturbation of the circular interface in $r=R$. The amplitude of the perturbation is $\zeta$. The central drop is the light blue disk of radius $r_{c}$.

$r=R$ regarding the separation temperature isotherm is not obvious and that the existence and the value of an interfacial tension in this zone can be subject to discussion. Furthermore, the interface driven toward the center sees its temperature rising up and so its interfacial tension, such that $\zeta<0$ implies $\Delta \gamma=\alpha \zeta>0$.

Following Nagel \& Gallaire [59] methodology, we solve the dimensionless Brinkman equation to get a dispersion relation of the instability. This equation is obtained provided a typical length scale $R$, a typical velocity $U$ and a typical pressure $P$ are introduced with $P=\mu_{2} U / R$ where $\mu_{2}$ the viscosity of the outer fluid. The dimensionless Brinkman equation writes:

$$
\eta_{j}\left(\Delta \overrightarrow{u_{j}}-k^{2} \overrightarrow{u_{j}}\right)-\vec{\nabla} p_{j}=0
$$

with $j=1$ for the inner fluid (the water-rich phase) and $j=2$ for the outer fluid (the homogeneous phase). The viscosity ratio $\eta_{j}$ is defined as $\eta_{1}=\mu_{1} / \mu_{2}=\eta$ and $\eta_{2}=1$. The parameter $k^{2}=12 R^{2} / H^{2}$, linked to the aspect ratio, comes from depth averaging (along $z$ direction). In this framework, the velocity $\overrightarrow{u_{j}}$ and the pressure $p_{j}$ fields are two-dimensional.

By introducing the stream function $\psi$ and by taking the curl of equation (10), the authors derive a more compact form:

$$
\left(\frac{\partial}{r \partial r} r \frac{\partial}{\partial r}+\frac{1}{r^{2}} \frac{\partial^{2}}{\partial \theta^{2}}\right)\left(\frac{\partial}{r \partial r} r \frac{\partial}{\partial r}+\frac{1}{r^{2}} \frac{\partial^{2}}{\partial \theta^{2}}-k^{2}\right) \psi=0
$$

The stream function is expanded as $\psi_{j}=\psi_{0 j}+\epsilon \psi_{1 j}$. The general solution for equation 11 gives:

$$
\begin{gathered}
\psi_{11}=i\left(a_{n} \frac{I_{n}(k r)}{I_{n}(k)}+b_{n} r^{n}\right) e^{i n \theta} \\
\psi_{12}=i\left(c_{n} \frac{K_{n}(k r)}{K_{n}(k)}+d_{n} r^{-n}\right) e^{i n \theta}
\end{gathered}
$$

with $I_{n}$ and $K_{n}$ the modified Bessel functions of first and second kind, which decay exponentially away from the interface. The parameters $a_{n}, b_{n}, c_{n}$, and $d_{n}$ have to be determined from the boundary conditions at the interface: impermeability, continuity of the normal and tangential velocity, and continuity of the normal and tangential stress.

As already discussed, the driving mechanism stems from the surface tension gradient which is the key difference with Nagel and Gallaire model. Under this assumption, the dimensionless normal and tangential boundary conditions of the interfacial stress are respectively:

$$
[\vec{n} \cdot \mathcal{T} \cdot \vec{n}]_{1}^{2}=\left(\frac{1}{C a}+\frac{\alpha \zeta}{\mu_{2} U}\right) R \mathcal{C}
$$




$$
[\vec{t} \cdot \mathcal{T} \cdot \vec{n}]_{1}^{2}=\vec{t} \cdot \vec{\nabla} \gamma \frac{R}{\mu_{2} U}
$$

where $C a=\mu_{2} U / \gamma_{0}$ is the capillary number, $\mathcal{C}$ is the curvature of the interface, $\vec{t} \cdot \vec{\nabla} \gamma=\alpha \frac{1}{R} \frac{\partial \zeta}{\partial \theta}$ is the linearised thermocapillary stress and $\mathcal{T}$ is the stress tensor. At first order in $\epsilon$, the tangential stress continuity equation becomes similar to the one of Nagel and Gallaire with an additional term on the right-hand side:

$$
\begin{array}{r}
\eta\left(2 a_{n} n^{2}+2 b_{n} n^{2}+a_{n} k^{2}-2 a_{n} k \frac{I_{n+1}(k)}{I_{n}(k)}-2 a_{n} n-2 b_{n} n\right) \\
-2 c_{n} n^{2}-2 d_{n} n^{2}-c_{n} k^{2}-2 c_{n} k \frac{K_{n+1}(k)}{K_{n}(k)}+2 c_{n} n-2 d_{n} n=n \frac{\alpha}{\mu_{2} U} \zeta
\end{array}
$$

In the same way, the normal stress jump at first order in $\epsilon$ writes:

$$
\begin{array}{r}
\eta\left(b_{n} k^{2}-2 a_{n} n-2 b_{n} n+2 a_{n} n k \frac{I_{n+1}(k)}{I_{n}(k)}+2 a_{n} n^{2}+2 b_{n} n^{2}\right)+d_{n} k^{2}+2 c_{n} n \\
+2 d_{n} n+2 c_{n} n k \frac{K_{n+1}(k)}{K_{n}(k)}-2 c_{n} n^{2}+2 d_{n} n^{2}=\frac{\pi}{4 C a} \zeta\left(n^{2}-1\right)+\frac{\alpha R}{\mu_{2} U} \zeta\left(\frac{\pi}{4}+\frac{2 R}{h}\right)
\end{array}
$$

Considering the full problem, the unknowns $a_{n}, b_{n}, c_{n}$ and $d_{n}$ are calculated and $\beta=\frac{1}{\zeta} \frac{\partial \zeta}{\partial t}$ can thus be determined, assuming that the aspect ratio $k$ and the capillary number $C a$ do not change significantly with time. The growth rate $\beta$ is given at the end of Appendix $B$.

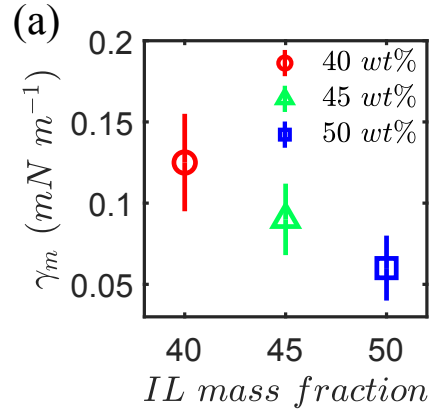

(b)

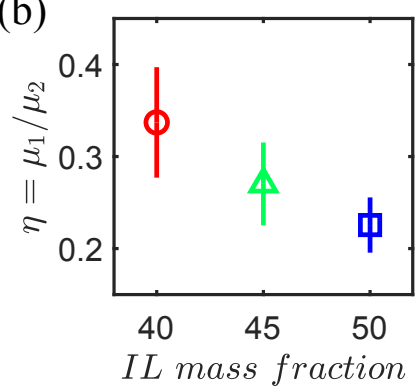

(c)
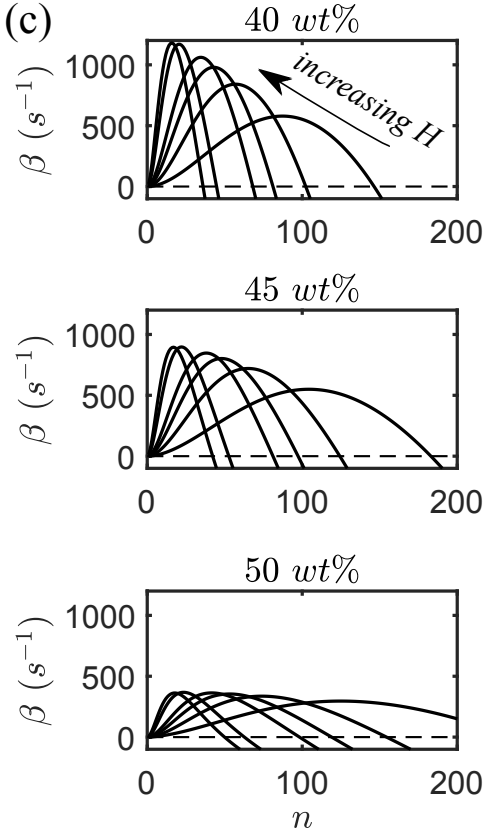

(d)

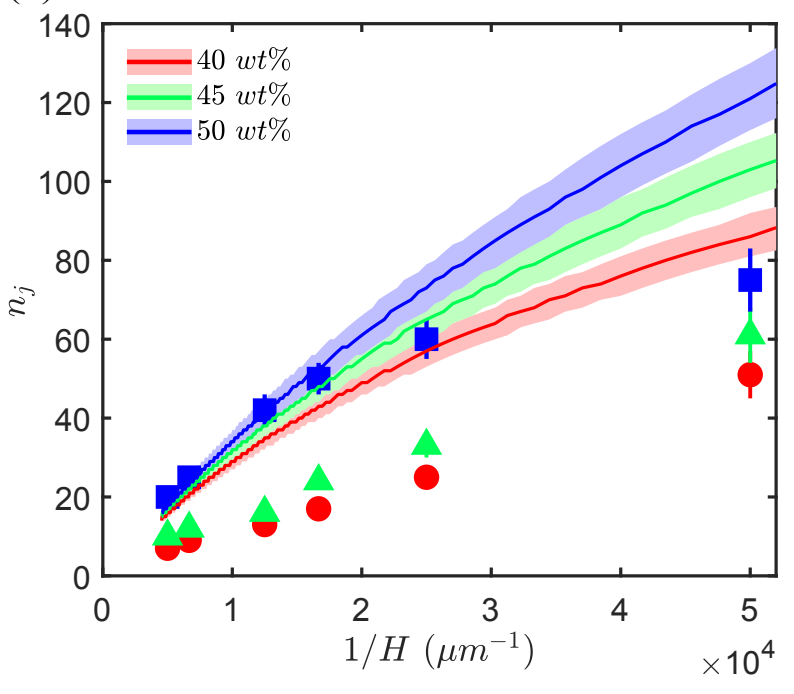

FIG. 10. (a) Mean interfacial tension along a jet for the three compositions. (b) Viscosity ratio between the two phases: the water-rich $\mu_{1}$ and the non-separated mixture $\mu_{2}$. (c) Growth rate $\beta$ calculated with the Brinkman model for all three compositions at six cavity heights: $20,40,60,80150$ and $200 \mu \mathrm{m}$. We use $d_{r} T=3000 \mathrm{~K}^{-\mathrm{m}^{-1}}$ and the values of $\gamma_{m}$ and $\eta$ from Figures 10(a) and (b). (d) Comparison between the number of jets measured and the prediction given by the Brinkman model. The shaded areas include all wavenumbers of the model where the growth rate is within $1 \%$ of the maximal growth rate.

The growth rate can be computed using the experimental values (temperature gradient, both surface tension gradient and viscosity as a function of IL mass fraction and temperature) detailed in Material $\&$ Methods. For the compositions of the solutions used in this section it is necessary to determine the values of the surface tension and viscosity to calculate the growth rate. Since the length of the Marangoni jets varies with the composition of the mixture, we evaluate a mean interfacial tension experienced by the jets in the temperature gradient. From the position of the jets a mean temperature can be extracted and a mean interfacial tension is deduced, plotted on Figure 10(a). The viscosity ratios of the two phases are plotted in Figure 10(b). These values are injected to numerically solve $\beta$. 
Figure 10(c) shows the growth rates for six cavity heights and three IL mass fraction with $d_{r} T=3 \mathrm{~K} \mathrm{~mm}^{-1}$. Remarkably, whatever the $H$ value and the mass fraction, the growth rate displays a range of unstable modes at large wavelength (small $n$ ). The only effect of the composition is a decreasing of the growth rate with increasing the IL mass fraction. From these curves the wavenumber of highest growth rate is extracted allowing to build this wavenumber as a function of the cavity height for different IL mass fraction. The sensitivity of the result is evidenced by varying $\beta$ by $1 \%$ as highlighted by the shaded area around the numerical value plotted in the figure $10(\mathrm{~d})$. We recover qualitatively the right tendencies yet the model overestimates the number of jets by a factor of two.

In fact, the linear stability analysis allows to obtain the wavelength at the very beginning of the instability process, i.e. the wavelength of the perturbation that can be observed at the interface in Figure 6 (red arrows at $3 \mathrm{~s}$ ). This wavelength can differ from the one of the fully developed pattern, which is stabilized by nonlinear effects that are not taken into account in the perturbation analysis. As the measurements are done on the fully developed pattern, discrepancies between measurements and prediction of the linear analysis can be expected. In particular, the radial extension of the cells $R-r_{c}$ appearing in the fully developed pattern does not emerge from the linear stability analysis. Considering this limitation, it is quite remarkable that we only have a factor of two difference if we take into account the extreme sensitivity of the model.

\section{IONIC LIQUID MASS FRACTION ABOVE 55\%}

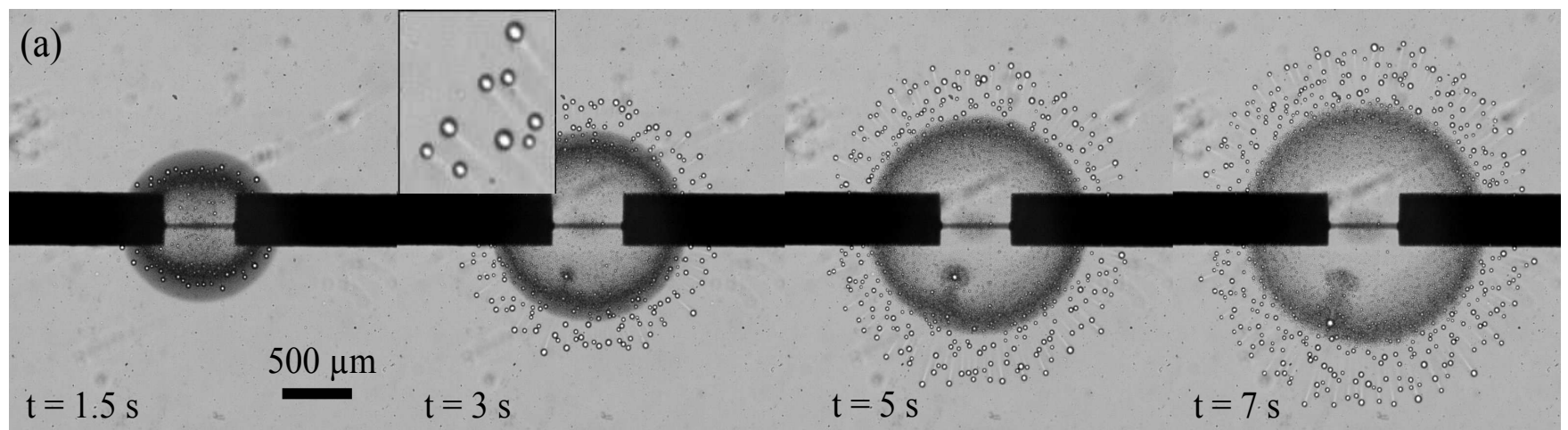

(b)

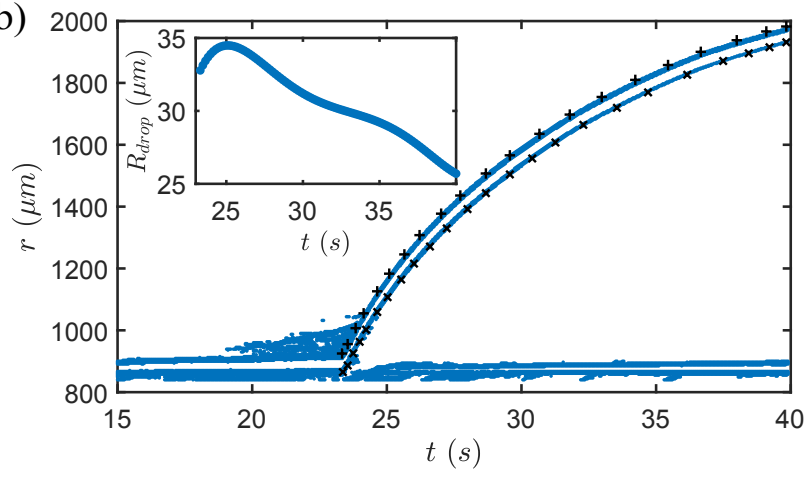

(c)

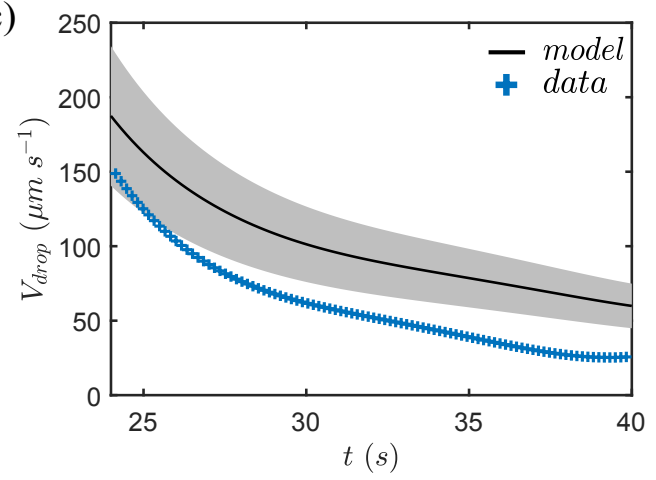

FIG. 11. (a) Time lapse of the phase separation dynamic for an IL mass fraction of $60 \%$. Above 55 wt $\%$ in IL, the water droplets nucleate and migrate radially outward because of thermocapillary actuation. As they go down the temperature gradient, their size shrinks as they redissolve and eventually vanish. The inset highlights the clear trails left behind the moving droplets as they release water-rich material in the IL-rich matrix. The voltage is 7 Volts $(80 \mathrm{~mW})$ and the cavity height is $H=80 \mu \mathrm{m}$. (b) Motion of a water drop in the temperature gradient. The drop is initially stuck at a surface. The black crosses give respectively the drop's front $(+)$ and rear $(\times)$. Inset: temporal evolution of the drop's radius. (c) Calculated and measured drop velocity versus time. We inject the temperature gradient values from Figure 2(b)inset in equation 18. The grey shaded area gives a $20 \%$ variation in the value of $d_{r} T$.

Above the IL weight fraction of $55 \%$ to $60 \%$ in IL, the water droplets become so scarce that the flower-like pattern is lost. The nucleating water droplets are sufficiently distant not to coalesce during their journey and they migrate outward in a radial motion under thermocapillary actuation, see Figure 11(a). The increasing speed of the drops with 
size is a noticeable feature of this effect. We emphasize that in this regime the water droplets are smaller than the cavity thickness, hence they do not experience any thermomechanical effect. Some drops may get pinned on surface defects, grow in size as the temperature rises in the cavity, and eventually detach when the thermocapillary forcing is strong enough. Their pace is slowing down as the gradient's slope flattens far from the hot point and their run ends shortly after crossing the critical temperature isotherm $T_{c} \approx 29^{\circ} \mathrm{C}$.

The drops also leave a clear trail behind them: their composition equilibrates as the temperature decreases and the excess of water released by the drops is conveyed at their rear by the interfacial convection, see inset in Figure 11(a). The nucleation events fade away after a few minutes as the temperature profile reaches steady state.

The thermocapillary motion of an initially pinned water droplet is shown on Figure 11(b). The droplet grows in size and detaches around $t=23.5 \mathrm{~s}$. Its radius, plotted on the inset, increases until $t=25 \mathrm{~s}$ and then decreases as the drop gets carried down the temperature gradient, away from the center. In order to validate the thermocapillary forcing, we compare on Figure 11(c) the velocity of the water drop to the prediction given by the following equation [62] (note here that the drop is considered in an infinite medium, that the Marangoni gradient is purely thermal and that the thermal conductivity of both phases are supposed equal):

$$
v_{\text {Tcap }}=\frac{R_{\text {drop }} d_{T} \gamma d_{r} T}{3 \eta_{I L}\left(1+3 \eta_{w} / 2 \eta_{I L}\right)}
$$

The drop moves slightly slower than the predicted velocity, however we recover the right trend and order of magnitude. Since this thermocapillary migration phenomena has been largely documented [63-65] and does not lead to a macroscopic phase separation of the binary mixture in our cavity, we do not further develop the analysis.

\section{CONCLUSION}

To conclude, we investigated the temperature gradient influence on the phase separation kinetics of LCST binary mixtures under confinement in which the volume fraction and wetting behavior of both constituents drive the dynamics. The thermal interfacial tension gradients are responsible for the flows, that oriented along the temperature gradient, drives the thin film drainage, the water droplet migration or the spontaneous digitation of one phase into the other. In all cases the resulting Marangoni strains behave as a pump accumulating the wetting phase in the warmer region and expelling the non-wetting phase in the cooler region. Thus, tuning the wall surface energy allows to select which component is concentrated in the center.

Three regimes are observed as summarized in the diagram of Figure 3 and have been rationalized. Briefly, concerning the possibility to separate the two phases, we observe that for IL mass fractions between 10 and $55 w t \%$ the IL-rich phase is concentrated in the warmer area, beyond $55 w t \%$ none of the two phases are selectively isolated.

In this article the regime that has attracted the most interest is the regime for which the IL mass fraction is between $35 \%$ and $55 \%$. In this regime, an instability develops that we have been able to explain as a Marangoni mechanism. A scaling analysis allows us to find the dependence of the number of jets $n_{j}$ with $H \times\left(R-r_{c}\right)$. We also derived a model based on Brinkman equations and we were able to find an agreement with the experimental results. In perspective, it would be interesting to look at the role of spatial wetting patterning [66-69]. We hope that this work will open the possibility of an alternative technique to the recycling of ionic liquids.

\section{ACKNOWLEDGMENTS}

The authors wish to thank B. Dollet for orienting us towards Nagel and Gallaire's paper. This work was supported by CNRS, ESPCI Paris, Agence Nationale de la Recherche (ANR) under the grant ANR-18-CE09-0029, IPGG (Equipex ANR-10-EQPX-34 and Labex ANR-10-LABX-31) and PSL (Idex ANR-10-IDEX-0001-02).

\section{APPENDIX A: LUTIDINE-WATER SYSTEM}

In order to tackle the reproducibility of the demixing pattern regarding the role of the wetting phase, we performed experiments using another LCST binary mixture widely employed in the literature: the lutidine-water system. The water-rich phase is expected to play the role of the wetting phase in a hydrophilic cavity, while the lutidine-rich phase wets preferentially a hydrophobic cavity [70]. Thus we performed the same experiments as previously with two 
layouts: a $20 w t \%$ lutidine solution in a hydrophilic cavity and a $40 w t \%$ lutidine solution in a hydrophobic cavity (the consolute point is around $28 w t \%$ in lutidine). Figure 12 displays the temporal evolution of the phase separation pattern for both cases, the lutidine-rich phase is made slightly darker by adding a dye. In the hydrophilic cavity the water-rich phase is accumulated in the central wetting drop whereas the lutidine-rich drops distributed around the perimeter give birth to the Marangoni jets. In the hydrophobic cavity the roles are swapped.

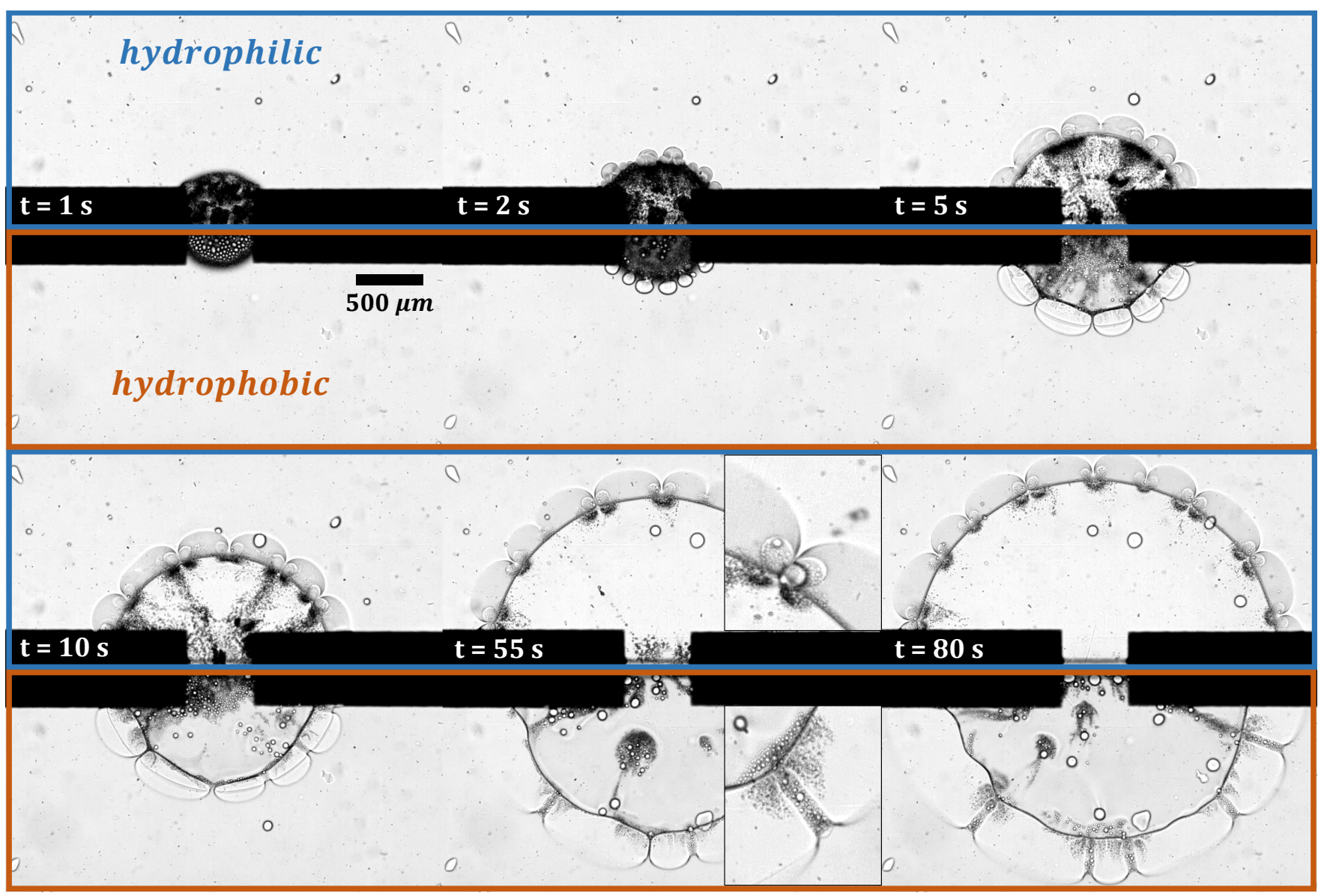

FIG. 12. Time lapse of the phase separation dynamic for two lutidine-water mixtures in two different cavities: $20 w t \%$ in lutidine for the hydrophilic cavity (oxygen plasma treated) and $40 w t \%$ in lutidine for the hydrophobic one (pristine PDMS cured at $130^{\circ} \mathrm{C}$ at least two hours). The lutidine-rich phase is slightly darker than the water-rich one. We recover the flower pattern formed by the periodic digitation. In the hydrophilic cavity the water-rich phase is accumulated at the center and the lutidine-rich phase gives the petals, while in the hydrophobic cavity roles are swapped. The voltage is $9.2 \mathrm{Volts}(135 \mathrm{~mW})$ and the cavity height is $H=60 \mu \mathrm{m}$. Insets: zoom on the Marangoni jets.

\section{APPENDIX B: STABILITY ANALYSIS}

Details on the stability analysis calculus are provided in this section. We recall that all the quantities are dimensionless.

The tangential $\vec{t}$ and normal $\vec{n}$ vectors to the interface (see Figure 9) write:

$$
\begin{aligned}
& \vec{t}=\left(1+\left(\frac{1}{R} \frac{\partial \zeta}{\partial \theta}\right)^{2}\right)^{-1 / 2}\left(\frac{1}{R} \frac{\partial \zeta}{\partial \theta} \overrightarrow{e_{r}}+\overrightarrow{e_{\theta}}\right) \approx \frac{1}{R} \frac{\partial \zeta}{\partial \theta} \overrightarrow{e_{r}}+\overrightarrow{e_{\theta}} \\
& \vec{n}=\left(1+\left(\frac{1}{R} \frac{\partial \zeta}{\partial \theta}\right)^{2}\right)^{-1 / 2}\left(\overrightarrow{e_{r}}-\frac{1}{R} \frac{\partial \zeta}{\partial \theta} \overrightarrow{e_{\theta}}\right) \approx \overrightarrow{e_{r}}-\frac{1}{R} \frac{\partial \zeta}{\partial \theta} \overrightarrow{e_{\theta}}
\end{aligned}
$$


In our configuration, the radius of the interface in the absence of perturbation $R_{0}$ grows slowly compared to the characteristic time of evolution of the perturbation $\zeta$. Consequently the unperturbed solution of our problem writes:

$$
u_{0 j}=v_{0 j}=0, \quad \psi_{0 j}=0 \quad \text { and }, \quad p_{0 j}=P_{j},
$$

with

$$
P_{1}-P_{2}=\frac{R C}{C a}=\frac{1}{C a}\left(\frac{\pi}{4}+\frac{2 R}{H}\right) .
$$

At the order $\epsilon$, the first terms $\psi_{1 j}$ of the expansion of the stream functions are given by eqs. (12) and (13) in which the constants $a_{n}, b_{n}, c_{n}, d_{n}$ need to be determined. Continuity of radial and tangential velocity at the interface write:

$$
\frac{\partial \psi_{11}}{\partial \theta}=\frac{\partial \psi_{12}}{\partial \theta} \text { and } \frac{\partial \psi_{11}}{\partial r}=\frac{\partial \psi_{12}}{\partial r}
$$

leading respectively to:

$$
a_{n}+b_{n}-c_{n}-d_{n}=0
$$

and to:

$$
a_{n} k \frac{I_{n+1}(k)}{I_{n}(k)}+c_{n} k \frac{K_{n+1}(k)}{K_{n}(k)}+n\left(a_{n}+b_{n}-c_{n}+d_{n}\right)=0
$$

At first order in $\epsilon$, the tangential stress continuity equation (eq. (15) of section ) becomes for $\psi_{1 j}$ :

$$
\left[\eta_{j}\left(r \frac{\partial}{\partial r}\left(\frac{\partial}{r \partial r} \psi_{1 j}\right)-\frac{\partial^{2}}{r^{2} \partial \theta^{2}} \psi_{1 j}\right)\right]_{1}^{2}=i n \frac{\alpha}{\mu_{2} U} \zeta
$$

yielding eq. (16):

$$
\begin{array}{r}
\eta\left(2 a_{n} n^{2}+2 b_{n} n^{2}+a_{n} k^{2}-2 a_{n} k \frac{I_{n+1}(k)}{I_{n}(k)}-2 a_{n} n-2 b_{n} n\right) \\
-2 c_{n} n^{2}-2 d_{n} n^{2}-c_{n} k^{2}-2 c_{n} k \frac{K_{n+1}(k)}{K_{n}(k)}+2 c_{n} n-2 d_{n} n=n \frac{\alpha}{\mu_{2} U} \tilde{\zeta}
\end{array}
$$

where $\tilde{\zeta}=\zeta_{0} e^{\beta t}$.

Taking into account the additional term due to thermocapillary stress, the normal stress jump writes:

$$
[\vec{n} \cdot \mathcal{T} \cdot \vec{n}]_{1}^{2}=\left(\frac{1}{C a}+\frac{\alpha \zeta}{\mu_{2} U}\right)\left(\frac{2 R}{H}+\frac{\pi}{4}\left(1-\left(1-n^{2}\right) \zeta\right)\right)
$$

where the $\pi / 4$ factor in the curvature term comes from the correction to the in-plane curvature in the case of perfect wetting [59]. At the first order in $\epsilon$, we obtain:

$$
\left[-p_{1 j}+2 \eta_{j} \frac{\partial}{\partial r}\left(\frac{1}{r} \frac{\partial \psi_{1 j}}{\partial \theta}\right)\right]_{1}^{2}=-\frac{\pi}{4 C a} \zeta\left(1-n^{2}\right)+\frac{\alpha R}{\mu_{2} U} \zeta\left(\frac{\pi}{4}+\frac{2 R}{H}\right)
$$

The pressure perturbation is integrated from the azimuthal part of the Brinkman equation as in [59]:

$$
p_{11}=\eta b_{n} r^{n} k^{2} e^{i n \theta} \quad \text { and } \quad p_{12}=-d_{n} r^{-n} k^{2} e^{i n \theta}
$$

Finally, the normal stress jump leads to:

$$
\begin{array}{r}
\eta\left(b_{n} k^{2}-2 a_{n} n-2 b_{n} n+2 a_{n} n k \frac{I_{n+1}(k)}{I_{n}(k)}+2 a_{n} n^{2}+2 b_{n} n^{2}\right)+d_{n} k^{2}+2 c_{n} n \\
+2 d_{n} n+2 c_{n} n k \frac{K_{n+1}(k)}{K_{n}(k)}-2 c_{n} n^{2}+2 d_{n} n^{2}=\frac{\pi}{4 C a} \tilde{\zeta}\left(n^{2}-1\right)+\frac{\alpha R}{\mu_{2} U} \tilde{\zeta}\left(\frac{\pi}{4}+\frac{2 R}{h}\right)
\end{array}
$$


Finally the kinematic condition at the interface (or impermeability condition) writes:

$$
\frac{\partial R}{\partial t}+\epsilon \frac{\partial \zeta}{\partial t}=u_{01}+\epsilon \frac{\partial u_{01}}{\partial r} \zeta+\epsilon u_{11}
$$

which yields at order $\epsilon$ :

$$
n\left(a_{n}+b_{n}\right)+\frac{\partial \tilde{\zeta}}{\partial t}=0
$$

Combining the boundary condition equations $23,24,16,17$ and 29 the unknowns $a_{n}, b_{n}, c_{n}, d_{n}$ and $\beta=\frac{1}{\tilde{\zeta}} \frac{\partial \tilde{\zeta}}{\partial t}$ can be determined. We display here the growth rate $\beta$ for wavenumber $n$ depending on the parameters of our system:

$$
\begin{aligned}
& \beta=-\left(n \left(8 \frac{\partial \gamma}{\partial r} \frac{I_{n+1}}{I_{n}} R^{2} k^{2}-16 \frac{\partial \gamma}{\partial r} \frac{I_{n+1}}{I_{n}} H n^{3}-32 \frac{\partial \gamma}{\partial r} \frac{I_{n+1}}{I_{n}} R^{2} n+4 \pi \frac{I_{n+1}}{I_{n}} \gamma H n+16 \frac{\partial \gamma}{\partial r} \frac{I_{n+1}}{I_{n}} \frac{K_{n+1}}{K_{n}} R^{2} k\right.\right. \\
& +32 \frac{\partial \gamma}{\partial r} \frac{I_{n+1}}{I_{n}} R^{2} \eta n+16 \frac{\partial \gamma}{\partial r} \frac{I_{n+1}}{I_{n}} \eta H n^{3}-4 \frac{\partial \gamma}{\partial r} \frac{I_{n+1}}{I_{n}} H k^{2} n-\pi \frac{I_{n+1}}{I_{n}} \gamma H k^{2}-4 \pi \frac{I_{n+1}}{I_{n}} \gamma H n^{3}-16 \frac{\partial \gamma}{\partial r} R^{2} \eta k n \\
& -8 \frac{\partial \gamma}{\partial r} \eta H k n^{2}+8 \frac{\partial \gamma}{\partial r} \frac{K_{n+1}}{K_{n}} R^{2} \eta k^{2}-2 \pi \eta \gamma H k n^{3}+\pi \frac{I_{n+1}}{I_{n}} \gamma H k^{2} n^{2}-4 \pi \frac{\partial \gamma}{\partial r} \frac{I_{n+1}}{I_{n}} R H n \\
& -2 \pi \frac{I_{n+1}}{I_{n}} \frac{K_{n+1}}{K_{n}} \gamma H k-4 \pi \frac{I_{n+1}}{I_{n}} \eta \gamma H n-16 \frac{\partial \gamma}{\partial r} \frac{I_{n+1}}{I_{n}} \frac{K_{n+1}}{K_{n}} R^{2} \eta k+\pi \frac{\partial \gamma}{\partial r} \frac{I_{n+1}}{I_{n}} R H k^{2}+2 \pi \eta \gamma H k n \\
& +8 \frac{\partial \gamma}{\partial r} \frac{I_{n+1}}{I_{n}} \frac{K_{n+1}}{K_{n}} H k n^{2}+4 \frac{\partial \gamma}{\partial r} \frac{K_{n+1}}{K_{n}} \eta H k^{2} n-\pi \frac{K_{n+1}}{K_{n}} \eta \gamma H k^{2}+4 \pi \frac{I_{n+1}}{I_{n}} \eta \gamma H n^{3}+\pi \frac{\partial \gamma}{\partial r} \frac{K_{n+1}}{K_{n}} R \eta H k^{2} \\
& -8 \frac{\partial \gamma}{\partial r} \frac{I_{n+1}}{I_{n}} \frac{K_{n+1}}{K_{n}} \eta H k n^{2}+2 \pi \frac{I_{n+1}}{I_{n}} \frac{K_{n+1}}{K_{n}} \gamma H k n^{2}+2 \pi \frac{\partial \gamma}{\partial r} \frac{I_{n+1}}{I_{n}} \frac{K_{n+1}}{K_{n}} R H k+\pi \frac{K_{n+1}}{K_{n}} \eta \gamma H k^{2} n^{2} \\
& +4 \pi \frac{\partial \gamma}{\partial r} \frac{I_{n+1}}{I_{n}} R \eta H n+2 \pi \frac{I_{n+1}}{I_{n}} \frac{K_{n+1}}{K_{n}} \eta \gamma H k-2 \pi \frac{\partial \gamma}{\partial r} R \eta H k n-2 \pi \frac{I_{n+1}}{I_{n}} \frac{K_{n+1}}{K_{n}} \eta \gamma H k n^{2} \\
& \left.\left.-2 \pi \frac{\partial \gamma}{\partial r} \frac{I_{n+1}}{I_{n}} \frac{K_{n+1}}{K_{n}} R \eta H k\right)\right) /\left(4 U H \mu \left(\frac{I_{n+1}}{I_{n}} k^{4}-8 \frac{I_{n+1}}{I_{n}} n^{2}+8 \frac{I_{n+1}}{I_{n}} n^{4}+\frac{K_{n+1}}{K_{n}} \eta^{2} k^{4}-8 \frac{I_{n+1}}{I_{n}} \eta^{2} n^{2}\right.\right. \\
& +8 \frac{I_{n+1}}{I_{n}} \eta^{2} n^{4}+4 \frac{I_{n+1}}{I_{n}} k^{2} n^{2}+8 \eta^{2} k n^{2}-8 \eta^{2} k n^{3}-2 \eta^{2} k^{3} n+2 \frac{I_{n+1}}{I_{n}} \frac{K_{n+1}}{K_{n}} k^{3}+\frac{I_{n+1}}{I_{n}} \eta k^{4}+\frac{K_{n+1}}{K_{n}} \eta k^{4} \\
& +16 \frac{I_{n+1}}{I_{n}} \eta n^{2}-16 \frac{I_{n+1}}{I_{n}} \eta n^{4}-8 \eta k n^{2}+8 \eta k n^{3}+2 \eta k^{3} n-4 \frac{I_{n+1}}{I_{n}} \frac{K_{n+1}}{K_{n}} k n^{3}-4 \frac{I_{n+1}}{I_{n}} \eta k^{2} n+4 \frac{K_{n+1}}{K_{n}} \eta k^{2} n \\
& -2 \frac{I_{n+1}}{I_{n}} \frac{K_{n+1}}{K_{n}} \eta^{2} k^{3}-4 \frac{I_{n+1}}{I_{n}} \eta k^{2} n^{2}+4 \frac{I_{n+1}}{I_{n}} \eta^{2} k^{2} n-4 \frac{K_{n+1}}{K_{n}} \eta k^{2} n^{2}-4 \frac{K_{n+1}}{K_{n}} \eta^{2} k^{2} n+4 \frac{I_{n+1}}{I_{n}} \frac{K_{n+1}}{K_{n}} k n \\
& \left.\left.+4 \frac{K_{n+1}}{K_{n}} \eta^{2} k^{2} n^{2}-8 \frac{I_{n+1}}{I_{n}} \frac{K_{n+1}}{K_{n}} \eta k n+4 \frac{I_{n+1}}{I_{n}} \frac{K_{n+1}}{K_{n}} \eta^{2} k n+8 \frac{I_{n+1}}{I_{n}} \frac{K_{n+1}}{K_{n}} \eta k n^{3}-4 \frac{I_{n+1}}{I_{n}} \frac{K_{n+1}}{K_{n}} \eta^{2} k n^{3}\right)\right)
\end{aligned}
$$

* marie-caroline.jullien@univ-rennes1.fr

[1] D. R. MacFarlane, A. L. Chong, M. Forsyth, M. Kar, R. Vijayaraghavan, A. Somers, and J. M. Pringle, New dimensions in salt-solvent mixtures: a 4th evolution of ionic liquids, Faraday Discussions 206, 9 (2017).

[2] H. Ohno, M. Yoshizawa-Fujita, and Y. Kohno, Functional design of ionic liquids: Unprecedented liquids that contribute to energy technology, bioscience, and materials sciences, Bulletin of the Chemical Society of Japan 92, 852 (2019).

[3] Y. Deguchi, N. Nakamura, and H. Ohno, Thermoresponsive ionic liquid/water mixtures for separation and purification technologies, Separation and Purification Technology 251, 117286 (2020).

[4] Y. Qiao, W. Ma, N. Theyssen, C. Chen, and Z. Hou, Temperature-responsive ionic liquids: fundamental behaviors and catalytic applications, Chemical Reviews 117, 6881 (2017).

[5] D. Dupont and K. Binnemans, Rare-earth recycling using a functionalized ionic liquid for the selective dissolution and revalorization of y 2 o 3: Eu 3+ from lamp phosphor waste, Green Chemistry 17, 856 (2015).

[6] D. Depuydt, L. Liu, C. Glorieux, W. Dehaen, and K. Binnemans, Homogeneous liquid-liquid extraction of metal ions with non-fluorinated bis (2-ethylhexyl) phosphate ionic liquids having a lower critical solution temperature in combination with water, Chemical Communications 51, 14183 (2015).

[7] B. Onghena, T. Opsomer, and K. Binnemans, Separation of cobalt and nickel using a thermomorphic ionic-liquid-based aqueous biphasic system, Chemical Communications 51, 15932 (2015).

[8] X. Fan, H. Liu, Y. Gao, Z. Zou, V. S. Craig, G. Zhang, and G. Liu, Forward-osmosis desalination with poly (ionic liquid) hydrogels as smart draw agents, Advanced Materials 28, 4156 (2016). 
[9] C.-H. Hsu, C. Ma, N. Bui, Z. Song, A. D. Wilson, R. Kostecki, K. M. Diederichsen, B. D. McCloskey, and J. J. Urban, Enhanced forward osmosis desalination with a hybrid ionic liquid/hydrogel thermoresponsive draw agent system, ACS Omega 4, 4296 (2019).

[10] E. Kamio, A. Takenaka, T. Takahashi, and H. Matsuyama, Fundamental investigation of osmolality, thermo-responsive phase diagram, and water-drawing ability of ionic-liquid-based draw solution for forward osmosis membrane process, Journal of Membrane Science 570, 93 (2019).

[11] Y. Cai, W. Shen, J. Wei, T. H. Chong, R. Wang, W. B. Krantz, A. G. Fane, and X. Hu, Energy-efficient desalination by forward osmosis using responsive ionic liquid draw solutes, Environmental Science: Water Research \& Technology 1, 341 (2015).

[12] A. Z. Haddad, A. K. Menon, H. Kang, J. J. Urban, R. S. Prasher, and R. Kostecki, Solar desalination using thermally responsive ionic liquids regenerated with a photonic heater, Environmental science \& technology 55, 3260 (2021).

[13] T. Araki and H. Tanaka, Hydrodynamic delocalization of phase separation in a locally cooled fluid mixture, EPL (Europhysics Letters) 65, 214 (2004).

[14] M. R. Moldover and J. W. Cahn, An interface phase transition: complete to partial wetting, Science 207, 1073 (1980).

[15] D. Beysens and D. Esteve, Adsorption phenomena at the surface of silica spheres in a binary liquid mixture, Physical Review Letters 54, 2123 (1985).

[16] S. Puri, Surface-directed spinodal decomposition, Journal of Physics: Condensed Matter 17, R101 (2005).

[17] H. Tanaka, Interplay between phase separation and wetting for a polymer mixture confined in a two-dimensional capillary: wetting-induced domain ordering and coarsening, EPL (Europhysics Letters) 24, 665 (1993).

[18] H. Tanaka, Dynamic interplay between phase separation and wetting in a binary mixture confined in a one-dimensional capillary, Physical Review Letters 70, 53 (1993).

[19] H. Tanaka, Wetting dynamics in a confined symmetric binary mixture undergoing phase separation, Physical Review Letters 70, 2770 (1993).

[20] H. Tanaka, Double phase separation in a confined, symmetric binary mixture: Interface quench effect unique to bicontinuous phase separation, Physical Review Letters 72, 3690 (1994).

[21] H. Tanaka and T. Sigehuzi, Spinodal decomposition of a symmetric binary fluid mixture in quasi two dimensions: Local orientational ordering of fluid tubes, Physical Review E 52, 829 (1995).

[22] K. Binder, Spinodal decomposition in confined geometry, Journal of Non Equilibrium Thermodynamics 23, 1 (1998).

[23] H. Tanaka, Interplay between wetting and phase separation in binary fluid mixtures: roles of hydrodynamics, Journal of Physics: Condensed Matter 13, 4637 (2001).

[24] L. Keiser, R. Herbaut, J. Bico, and E. Reyssat, Washing wedges: capillary instability in a gradient of confinement, Journal of Fluid Mechanics 790, 619 (2016).

[25] A. Cazabat, F. Heslot, S. Troian, and P. Carles, Fingering instability of thin spreading films driven by temperature gradients, Nature 346, 824 (1990).

[26] M. Dietzel and S. M. Troian, Mechanism for spontaneous growth of nanopillar arrays in ultrathin films subject to a thermal gradient, Journal of Applied Physics 108, 074308 (2010).

[27] V. Miralles, B. Selva, I. Cantat, and M.-C. Jullien, Foam drainage control using thermocapillary stress in a two-dimensional microchamber, Physical Review etters 112, 238302 (2014).

[28] G. Koleski, A. Vilquin, J.-C. Loudet, T. Bickel, and B. Pouligny, Azimuthal instability of the radial thermocapillary flow around a hot bead trapped at the water-air interface, Physics of Fluids 32, 092108 (2020).

[29] N. Young, J. S. Goldstein, and M. J. Block, The motion of bubbles in a vertical temperature gradient, Journal of Fluid Mechanics 6, 350 (1959).

[30] M. Assenheimer, B. Khaykovich, and V. Steinberg, Phase separation of a critical binary mixture subjected to a temperature gradient, Physica A: Statistical Mechanics and its Applications 208, 373 (1994).

[31] F. Morick and D. Woermann, Convection generated by a gradient of interfacial tension in a phase separated binary liquid mixture with a miscibility gap, Zeitschrift für Physikalische Chemie 205, 1 (1998).

[32] A. Würger, Thermally driven marangoni surfers, arXiv preprint arXiv:1407.6818 (2014).

[33] T. Araki and A. Maciołek, Illumination-induced motion of a janus nanoparticle in binary solvents, Soft Matter 15, 5243 (2019).

[34] J. R. Gomez-Solano, S. Roy, T. Araki, S. Dietrich, and A. Maciołek, Transient coarsening and the motility of optically heated janus colloids in a binary liquid mixture, Soft Matter 16, 8359 (2020).

[35] T. Zinn, L. Sharpnack, and T. Narayanan, Phoretic dynamics of colloids in a phase separating critical liquid mixture, Physical Review Research 2, 033177 (2020).

[36] K. Dietrich, N. Jaensson, I. Buttinoni, G. Volpe, and L. Isa, Microscale marangoni surfers, Physical Review Letters 125, 098001 (2020).

[37] D. Beysens, Y. Garrabos, V. Nikolayev, C. Lecoutre-Chabot, J.-P. Delville, and J. Hegseth, Liquid-vapor phase separation in a thermocapillary force field, EPL (Europhysics Letters) 59, 245 (2002).

[38] Although for this latter work the authors have corrected their interpretation since then: the separation mechanism is not driven by Marangoni forces. In the case of a pure fluid undergoing phase transition, the vapor-liquid interface is expected to remain isothermal at all time thus thermocapillary (Marangoni) motion should not exist. Instead the migration is imputed to an asymmetric evaporation-condensation mechanism along the droplet interface [71-73].

[39] M. Pascual, A. Poquet, A. Vilquin, and M.-C. Jullien, Phase separation of an ionic liquid mixture assisted by a temperature gradient, Physical Review Fluids 6, 024001 (2021).

[40] E. D. Siggia, Late stages of spinodal decomposition in binary mixtures, Physical review A 20, 595 (1979). 
[41] D. A. Beysens, Kinetics and morphology of phase separation in fluids: The role of droplet coalescence, Physica A: Statistical Mechanics and its Applications 239, 329 (1997).

[42] Y. Kohno, H. Arai, S. Saita, and H. Ohno, Material design of ionic liquids to show temperature-sensitive lcst-type phase transition after mixing with water, Australian Journal of Chemistry 64, 1560 (2012).

[43] A. Nitta, T. Morita, K. Nishikawa, and Y. Koga, Mixing scheme of an aqueous solution of tetrabutylphosphonium trifluoroacetate in the water-rich region, Physical Chemistry Chemical Physics 19, 16888 (2017).

[44] M. Kerdraon, J. D. McGraw, B. Dollet, and M.-C. Jullien, Self-similar relaxation of confined microfluidic droplets, Physical Review Letters 123, 024501 (2019).

[45] H. Tanaka, Viscoelastic phase separation, Journal of Physics: Condensed Matter 12, R207 (2000).

[46] See Supplemental Material at [URL will be inserted by publisher] for the movie showing the phase separation in both three regimes..

[47] B. Selva, I. Cantat, and M.-C. Jullien, Temperature-induced migration of a bubble in a soft microcavity, Physics of Fluids 23, 052002 (2011).

[48] R. De Dier, W. Sempels, J. Hofkens, and J. Vermant, Thermocapillary fingering in surfactant-laden water droplets, Langmuir 30, 13338 (2014).

[49] L. Keiser, H. Bense, P. Colinet, J. Bico, and E. Reyssat, Marangoni bursting: evaporation-induced emulsification of binary mixtures on a liquid layer, Physical Review Letters 118, 074504 (2017).

[50] F. Wodlei, J. Sebilleau, J. Magnaudet, and V. Pimienta, Marangoni-driven flower-like patterning of an evaporating drop spreading on a liquid substrate, Nature Communications 9, 1 (2018).

[51] P. Marmottant, F. Orthion, and S. Rafaï, When dipping toast into a cup of tea leads to a scientific investigation, American Journal of Physics 87, 950 (2019).

[52] X. Ma, M. Zhong, Y. He, Z. Liu, and Z. Li, Fingering instability in marangoni spreading on a deep layer of polymer solution, Physics of Fluids 32, 112112 (2020).

[53] H. Tanaka and T. Araki, Spontaneous double phase separation induced by rapid hydrodynamic coarsening in twodimensional fluid mixtures, Physical Review Letters 81, 389 (1998).

[54] T. Sigehuzi and H. Tanaka, Coarsening mechanism of phase separation caused by a double temperature quench in an off-symmetric binary mixture, Physical Review E 70, 051504 (2004).

[55] R. Krechetnikov, Structure of marangoni-driven singularities, Physics of Fluids 24, 022111 (2012).

[56] A. Girot, J. Petit, R. Saiseau, T. Guérin, H. Chraibi, U. Delabre, and J.-P. Delville, Conical interfaces between two immiscible fluids induced by an optical laser beam, Physical Review Letters 122, 174501 (2019).

[57] H. Chesneau, J. Petit, H. Chraïbi, and J.-P. Delville, Dynamics and flow characterization of liquid fountains produced by light scattering, Physical Review Fluids 5, 024002 (2020).

[58] See Supplemental Material at [URL will be inserted by publisher] for the movie showing the spontaneous generation of new jets..

[59] M. Nagel and F. Gallaire, A new prediction of wavelength selection in radial viscous fingering involving normal and tangential stresses, Physics of Fluids 25, 124107 (2013).

[60] F. Charru, Hydrodynamic instabilities, Vol. 37 (Cambridge University Press, 2011).

[61] S. H. Davis, Thermocapillary instabilities, Annual Review of Fluid Mechanics 19, 403 (1987).

[62] J. Chen and K. J. Stebe, Surfactant-induced retardation of the thermocapillary migration of a droplet, Journal of Fluid Mechanics 340, 35 (1997).

[63] B. Braun, C. Ikier, H. Klein, and D. Woermann, Thermocapillary migration of droplets in a binary mixture with miscibility gap during liquid/liquid phase separation under reduced gravity, Journal of Colloid and Interface Science 159, 515 (1993).

[64] B. Braun, H. Klein, and D. Woermann, Thermocapillary motion of droplets of a phase separated binary liquid mixture with a closed loop miscibility gap, Zeitschrift für Physikalische Chemie 193, 175 (1996).

[65] H. Klein, B. Braun, C. Ikier, and D. Woermann, Phase separation induced by marangoni convection, Advances in Space Research 22, 1245 (1998).

[66] M. Pascual, M. Kerdraon, Q. Rezard, M.-C. Jullien, and L. Champougny, Wettability patterning in microfluidic devices using thermally-enhanced hydrophobic recovery of pdms, Soft Matter 15, 9253 (2019).

[67] P. Das, P. K. Jaiswal, and S. Puri, Surface-directed spinodal decomposition on chemically patterned substrates, Physical Review E 102, 012803 (2020).

[68] P. Das, P. K. Jaiswal, and S. Puri, Surface-directed spinodal decomposition on morphologically patterned substrates, Physical Review E 102, 032801 (2020).

[69] G. Guyard, A. Vilquin, N. Sanson, S. Jouenne, F. Restagno, and J. D. McGraw, Near-surface rheology and hydrodynamic boundary condition of semi-dilute polymer solutions, Soft Matter 17, 3765 (2021).

[70] D. Beysens and T. Narayanan, Wetting-induced aggregation of colloids, Journal of Statistical Physics 95, 997 (1999).

[71] A. Onuki and K. Kanatani, Droplet motion with phase change in a temperature gradient, Physical Review E 72, 066304 (2005).

[72] A. Onuki, Bubble and droplet motion in binary mixtures: Evaporation-condensation mechanism and marangoni effect, Physical Review E 79, 046311 (2009).

[73] V. S. Nikolayev, Y. Garrabos, C. Lecoutre, G. Pichavant, D. Chatain, and D. Beysens, Evaporation condensation-induced bubble motion after temperature gradient set-up, Comptes Rendus Mécanique 345, 35 (2017). 